\title{
Branched-chain amino acid metabolism is regulated by ERRa in primary human myotubes and is further impaired by glucose loading in type 2 diabetes
}

\author{
Rasmus J. O. Sjögren ${ }^{1}$ - David Rizo-Roca ${ }^{1}$ - Alexander V. Chibalin ${ }^{1}$ • Elin Chorell ${ }^{2} \cdot$ Regula Furrer $^{3}$. \\ Shintaro Katayama ${ }^{4}$. Jun Harada ${ }^{5}$. Håkan K. R. Karlsson ${ }^{1}$. Christoph Handschin ${ }^{3}$. Thomas Moritz ${ }^{6}$. Anna Krook ${ }^{7}$. \\ Erik Näslund $^{8}$ • Juleen R. Zierath ${ }^{1,7}$ (iD
}

Received: 17 January 2021 / Accepted: 12 March 2021 / Published online: 16 June 2021

(C) The Author(s) 2021

\begin{abstract}
Aims/hypothesis Increased levels of branched-chain amino acids (BCAAs) are associated with type 2 diabetes pathogenesis. However, most metabolomic studies are limited to an analysis of plasma metabolites under fasting conditions, rather than the dynamic shift in response to a metabolic challenge. Moreover, metabolomic profiles of peripheral tissues involved in glucose homeostasis are scarce and the transcriptomic regulation of genes involved in BCAA catabolism is partially unknown. This study aimed to identify differences in circulating and skeletal muscle BCAA levels in response to an OGTT in individuals with normal glucose tolerance (NGT) or type 2 diabetes. Additionally, transcription factors involved in the regulation of the BCAA gene set were identified.

Methods Plasma and vastus lateralis muscle biopsies were obtained from individuals with NGT or type 2 diabetes before and after an OGTT. Plasma and quadriceps muscles were harvested from skeletal muscle-specific Ppargcla knockout and transgenic mice. BCAA-related metabolites and genes were assessed by LC-MS/MS and quantitative RT-PCR, respectively. Small interfering RNA and adenovirus-mediated overexpression techniques were used in primary human skeletal muscle cells to study the role of PPARGC1A and ESRRA in the expression of the BCAA gene set. Radiolabelled leucine was used to analyse the impact of oestrogen-related receptor $\alpha(\mathrm{ERR} \alpha)$ knockdown on leucine oxidation.

Results Impairments in BCAA catabolism in people with type 2 diabetes under fasting conditions were exacerbated after a glucose load. Branched-chain keto acids were reduced 37-56\% after an OGTT in the NGT group, whereas no changes were detected in individuals with type 2 diabetes. These changes were concomitant with a stronger correlation with glucose homeostasis biomarkers and downregulated expression of branched-chain amino acid transaminase 2, branched-chain keto acid dehydrogenase complex subunits and $69 \%$ of downstream BCAA-related genes in skeletal muscle. In primary human myotubes overexpressing peroxisome proliferator-activated receptor $\gamma$ coactivator- $1 \alpha$ (PGC- $1 \alpha$, encoded by PPARGC1A), $61 \%$ of the analysed BCAA genes were upregulated, while $67 \%$ were downregulated in the quadriceps of skeletal muscle-specific Ppargcla knockout mice. ESRRA (encoding ERR $\alpha$ ) silencing completely abrogated the PGC-1 $\alpha$-induced upregulation of BCAA-related genes in primary human myotubes.
\end{abstract}

Rasmus J. O. Sjögren and David Rizo-Roca contributed equally.

Juleen R. Zierath

juleen.zierath@ki.se

1 Department of Molecular Medicine and Surgery, Integrative Physiology, Karolinska Institutet, Stockholm, Sweden

2 Department of Public Health and Clinical Medicine, Umeå University, Umeå, Sweden

3 Biozentrum, University of Basel, Basel, Switzerland

4 Department of Biosciences and Nutrition, Karolinska Institutet, Huddinge, Sweden
5 Cardiovascular-Metabolics Research Laboratories, Daiichi Sankyo Co., Ltd., Tokyo, Japan

6 Swedish Metabolomics Centre, Department of Forest Genetics and Plant Physiology, Swedish University of Agricultural Sciences, Umeå, Sweden

7 Department of Physiology and Pharmacology, Integrative Physiology, Karolinska Institutet, Stockholm, Sweden

8 Division of Surgery, Department of Clinical Sciences, Danderyd Hospital, Karolinska Institutet, Stockholm, Sweden 


\section{Research in context}

\section{What is already known about this subject?}

- Circulating levels of branched-chain amino acids (BCAA) are elevated in type 2 diabetes

- Peroxisome proliferator-activated receptor $\gamma$ coactivator-1a (PGC-1a) is involved in the transcription of the BCAA gene set

What is the key question?

- Does metabolic inflexibility associated with type 2 diabetes encompass BCAA homeostasis and PGC-1 a-mediated transcription of the BCAA gene set?

\section{What are the new findings?}

- BCAA homeostasis is further compromised by a glucose challenge in type 2 diabetes

- An OGTT reveals coordinated regulation between BCAA metabolites, blood glucose and $\mathrm{Hb} \mathrm{A}_{1 c}$ levels

- Oestrogen-related receptor a (ERRa) is essential for PGC-1a-mediated BCAA gene expression in primary human myotubes

\section{How might this impact on clinical practice in the foreseeable future?}

- $\quad$ An OGTT can be used to underscore impairments in BCAA metabolism. These findings suggest that interventions targeting the PGC-1a-ERRa axis may improve BCAA homeostasis

Conclusions/interpretation Metabolic inflexibility in type 2 diabetes impacts BCAA homeostasis and attenuates the decrease in circulating and skeletal muscle BCAA-related metabolites after a glucose challenge. Transcriptional regulation of BCAA genes in primary human myotubes via PGC- $1 \alpha$ is ERR $\alpha$-dependent.

Keywords Branched-chain amino acid - Oestrogen-related receptor $\alpha$. Oral glucose tolerance test · Peroxisome proliferator-activated receptor $\gamma$ coactivator $1-\alpha \cdot$ Skeletal muscle $\cdot$ Type 2 diabetes

\begin{tabular}{|c|c|}
\hline \\
\hline \multicolumn{2}{|c|}{$\begin{array}{l}\text { Abbreviations } \\
\begin{array}{ll}\text { Ad-PGC1A Adenoviral vector for human or } \\
\\
\end{array} \quad \text { mouse PPARGC1A }\end{array}$} \\
\hline Ad-GFP & Adenoviral vector for $g f p$ \\
\hline BCAA & Branched-chain amino acid \\
\hline BCAT2 & Branched-chain amino acid transaminase 2 \\
\hline BCKA & Branched-chain $\alpha$-keto acid \\
\hline $\mathrm{BCKDH}$ & Branched-chain keto acid dehydrogenase \\
\hline BCKDHA & $\begin{array}{l}\text { Branched-chain } \alpha \text {-keto acid } \\
\text { dehydrogenase E1 subunit } \alpha\end{array}$ \\
\hline BCKDK & $\begin{array}{l}\text { Branched-chain keto acid } \\
\text { dehydrogenase kinase }\end{array}$ \\
\hline BT2 & $\begin{array}{l}\text { 3,6-Dichlorobenzo(b)thiophene- } \\
\text { 2-carboxylic acid }\end{array}$ \\
\hline $\operatorname{ERR} \alpha$ & Oestrogen-related receptor $\alpha$ \\
\hline 3-HIB & 3-Hydroxyisobutyrate \\
\hline HSMC & Human skeletal muscle cell \\
\hline $\mathrm{mKO}$ & $\begin{array}{l}\text { Ppargcla muscle-specific } \\
\text { knockout mice }\end{array}$ \\
\hline mTG & $\begin{array}{l}\text { Skeletal muscle-specific Ppargcla } \\
\text { transgenic mice }\end{array}$ \\
\hline NGT & Normal glucose tolerance \\
\hline
\end{tabular}

$\begin{array}{ll}\text { PGC-1 } \alpha & \begin{array}{l}\text { Peroxisome proliferator-activated } \\ \text { receptor } \gamma \text { coactivator- } 1 \alpha\end{array} \\ \text { PPAR } \gamma & \begin{array}{l}\text { Peroxisome proliferator-activated receptor } \gamma \\ \text { PPM1K }\end{array} \\ & \begin{array}{l}\text { Protein phosphatase, } \\ \mathrm{Mg}^{2+} / \mathrm{Mn}^{2+} \text {-dependent } 1 \mathrm{~K}\end{array} \\ \text { SiRNA } & \text { Small interfering RNA } \\ \text { WT } & \text { Wild-type }\end{array}$

\section{Introduction}

Type 2 diabetes is a metabolic disease characterised by chronic hyperglycaemia and insulin resistance [1]. These metabolic derangements severely affect pathways controlling the appropriate sensing and handling of nutrients, thereby leading to a positive energy balance and metabolic inflexibility, which further compromises whole-body glucose homeostasis [2]. Overnutrition and type 2 diabetes-related disturbances also affect non-glucose metabolites such as branched-chain amino acids (BCAAs; leucine, isoleucine and valine) [3]; the 
utilisation and metabolism of these essential amino acids are exquisitely regulated in healthy individuals. While BCAAs and related metabolites play a role in protein synthesis, they also modulate liver gluconeogenesis and lipogenesis rates, cell growth and nutrient signalling, and can enter the tricarboxylic acid cycle to produce energy [4]. Under pathological conditions, BCAAs, especially in a context of overnutrition, disrupt insulin sensitivity and secretion [5].

Circulating levels of BCAAs are elevated in individuals with obesity, insulin resistance and/or type 2 diabetes [6]. Metabolomic profiling of blood metabolites has revealed a signature of altered BCAA catabolism in obese individuals, strongly associated with insulin resistance [7]. BCAA-related metabolites are predictive of type 2 diabetes pathogenesis [8] as well as being prognostic markers for intervention outcomes [9, 10]. Mendelian randomisation analysis suggested a causal link between genetic variants associated with impaired BCAA catabolism and higher risk of type 2 diabetes [11]. Therefore, there is growing evidence that high levels of BCAAs and related intermediate metabolites are not only type 2 diabetes biomarkers but also pathophysiological factors. However, many of these studies were conducted in individuals after an overnight fast, under conditions in which protein degradation in skeletal muscle and a concomitant release of amino acids to support gluconeogenesis [12] could mask deeper alterations in BCAA homeostasis. Moreover, these studies do not provide insight into the dynamic shift in metabolism that occurs in response to nutritional challenges. An analysis of metabolomic signatures in fasted individuals with glucose tolerance or type 2 diabetes before and after a glucose challenge may provide insight into dynamic changes in BCAAs and related metabolites and the consequences of insulin resistance.

Skeletal muscle is the largest contributor to systemic BCAA oxidation [13] and therefore impairments in glucose and BCAA metabolism in myocytes have an impact on whole-body metabolic homeostasis. While BCAA-related gene expression and oxidation rates are reduced in vastus lateralis muscle from individuals with insulin resistance [14], studies of BCAA metabolism in human skeletal muscle are scarce. BCAA catabolism occurs in the mitochondrial matrix, implying that alterations in mitochondrial proteins may influence BCAA metabolism. In transgenic mice overexpressing the mitochondrial biogenesis inducer peroxisome proliferatoractivated receptor $\gamma(\operatorname{PPAR} \gamma)$ coactivator- $1 \alpha(\mathrm{PGC}-1 \alpha)$, BCAA levels are reduced in gastrocnemius muscle [15]. Conversely, administration of the $\operatorname{PPAR} \gamma$ agonist thiazolidinedione rosiglitazone improves glycaemic control and increases circulating levels of BCAAs in individuals with type 2 diabetes [16]. Nevertheless, mechanisms underlying the direct role of PGC- $1 \alpha$ in the regulation of BCAA catabolism are unclear.

The aim of this study was to assess whether an OGTT can further reveal an impairment in BCAA metabolism in individuals with type 2 diabetes. We also aimed to identify a PGC- $1 \alpha$ transcriptional partner in the regulation of the BCAA gene program by using cell and mouse models in which PGC$1 \alpha$ expression was altered.

\section{Methods}

Participants Thirty-two men with normal glucose tolerance (NGT) and 29 men with type 2 diabetes aged 44-69 years were recruited to participate in this study. All participants gave their informed consent. The study was approved by the regional ethical review board in Stockholm. The experimental procedures were conducted according to the Declaration of Helsinki. Participants underwent a clinical health screening consisting of clinical chemistry and anthropometric measurements (Tables 1, 2). Five individuals in the control group that exhibited impaired glucose tolerance and one individual diagnosed with type 2 diabetes that exhibited NGT were excluded from the study (Fig. 1). Individuals with type 2 diabetes had higher blood glucose, $\mathrm{HbA}_{1 \mathrm{c}}$ and HOMA-IR, as well as higher BMI and waist circumference than individuals with NGT (Tables 1 and 2). Individuals with type 2 diabetes included in the transcriptomic analysis were older than the control group of individuals with NGT (Table 2). Individuals with type 2 diabetes were treated with metformin ( $n=25$; daily dose range $500-3000 \mathrm{mg}$ ) and/or sulfonylurea (glibenclamide [glyburide], $n=5,2-5 \mathrm{mg}$ daily; glimepiride, $n=1,3 \mathrm{mg}$ daily; glipizide, $n=1,5 \mathrm{mg}$ daily). Glucoselowering medication was taken by the individuals with type 2 diabetes after the skeletal muscle biopsy collection.

Participants arrived at the clinic at 07:45 h after a $12 \mathrm{~h}$ fast. A catheter was inserted into an arm vein and fasting blood samples were obtained. After applying local anaesthesia subcutaneously (lidocaine hydrochloride $5 \mathrm{mg} / \mathrm{ml}$; Aspen Nordic, Denmark), biopsies from the vastus lateralis were obtained using a conchotome (AgnTho's, Sweden). After $\sim 15 \mathrm{~min}$, participants ingested a solution containing $75 \mathrm{~g}$ of glucose and underwent an OGTT. A blood sample was obtained $30 \mathrm{~min}$ after glucose ingestion. After $120 \mathrm{~min}$, a blood sample and vastus lateralis biopsy were obtained. All biopsies were immediately snap-frozen in liquid nitrogen and stored at $-80^{\circ} \mathrm{C}$. Metabolomic analysis was performed by Metabolon (Durham, NC, USA) as described [17]. See electronic supplementary material (ESM) Methods: Human plasma and skeletal muscle metabolomics for details.

Cell culture experiments Human satellite cells were harvested from vastus lateralis skeletal muscle biopsies of healthy volunteers from both sexes and differentiated as described [18]. Mouse C2C12 myoblasts (ATCC CRL-1772; ATCC, VA, USA) were propagated in growth media (DMEM, 20\% FBS and $1 \%$ antibiotic-antimycotic (11570486; Fisher 
Table 1 Clinical characteristics of the participants included in the metabolomic analysis

\begin{tabular}{|c|c|c|c|}
\hline Characteristic & NGT & Type 2 diabetes & $p$ value \\
\hline Age (years) & $59 \pm 2.4$ & $62 \pm 1.2$ & 0.1611 \\
\hline Weight (kg) & $81 \pm 2.7$ & $88 \pm 1.8$ & 0.0209 \\
\hline Height (m) & $1.77 \pm 0.02$ & $1.79 \pm 0.01$ & 0.4082 \\
\hline BMI $\left(\mathrm{kg} / \mathrm{m}^{2}\right)$ & $25.95 \pm 0.59$ & $27.66 \pm 0.47$ & 0.0307 \\
\hline Waist (cm) & $90 \pm 1.6$ & $100 \pm 1.6$ & 0.0002 \\
\hline Hip (cm) & $97 \pm 1.2$ & $101 \pm 1.2$ & 0.0241 \\
\hline WHR & $0.93 \pm 0.01$ & $0.99 \pm 0.01$ & 0.0008 \\
\hline Pulse (beats/min) & $62 \pm 2.0$ & $68 \pm 1.6$ & 0.0234 \\
\hline Fasting plasma glucose (mmol/l) & $5.41 \pm 0.13$ & $8.59 \pm 0.37$ & $<0.0001$ \\
\hline $120 \mathrm{~min}$ plasma glucose $(\mathrm{mmol} / \mathrm{l})$ & $6.05 \pm 0.25$ & $16.66 \pm 0.58$ & $<0.0001$ \\
\hline $\mathrm{HbA}_{1 \mathrm{c}}(\mathrm{mmol} / \mathrm{mol})$ & $36.13 \pm 1.13$ & $51.19 \pm 1.32$ & $<0.0001$ \\
\hline $\mathrm{HbA}_{1 \mathrm{c}}(\%)$ & $5.5 \pm 0.10$ & $6.8 \pm 0.12$ & $<0.0001$ \\
\hline Serum insulin (pmol/l) & $52.37 \pm 5.30$ & $85.51 \pm 9.06$ & 0.0124 \\
\hline 120 min serum insulin $(\mathrm{pmol} / \mathrm{l})$ & $294.2 \pm 43.41$ & $327.5 \pm 42.06$ & 0.6155 \\
\hline Plasma creatinine $(\mu \mathrm{mol} / \mathrm{l})$ & $84.53 \pm 2.65$ & $80.72 \pm 2.17$ & 0.2794 \\
\hline Plasma AST $(\mu k a t / l)$ & $0.42 \pm 0.04$ & $0.39 \pm 0.03$ & 0.5992 \\
\hline Plasma ALT ( $\mu$ kat/l) & $0.38 \pm 0.06$ & $0.48 \pm 0.04$ & 0.1161 \\
\hline Plasma triacylglycerol (mmol/l) & $1.08 \pm 0.14$ & $1.41 \pm 0.13$ & 0.1093 \\
\hline Plasma cholesterol (mmol/1) & $5.27 \pm 0.17$ & $4.50 \pm 0.14$ & 0.0014 \\
\hline Plasma HDL-cholesterol (mmol/l) & $1.33 \pm 0.06$ & $1.24 \pm 0.06$ & 0.3562 \\
\hline Plasma LDL-cholesterol (mmol/l) & $3.47 \pm 0.13$ & $2.62 \pm 0.14$ & 0.0003 \\
\hline Serum C-peptide (nmol/l) & $0.69 \pm 0.04$ & $0.99 \pm 0.08$ & 0.0115 \\
\hline HOMA-IR & $1.71 \pm 0.22$ & $5.28 \pm 0.65$ & 0.0013 \\
\hline
\end{tabular}

Results are mean \pm SEM for individuals with NGT $(n=15)$ and type 2 diabetes $(n=26)$

Statistical analysis was performed using Student's $t$ test

ALT, alanine aminotransferase; AST, aspartate aminotransferase
Scientific, Sweden). After $\sim 12 \mathrm{~h}$, medium was changed to differentiation medium (DMEM, 2\% horse serum and 1\% antibiotic-antimycotic) and differentiated myotubes were cultured for 6 days before the final experiments. For pharmacological inhibition of oestrogen-related receptor $\alpha($ ERR $\alpha)$, human skeletal muscle cells (HSMCs) were incubated with $5 \mu \mathrm{mol} / \mathrm{l}$ of XCT-790 (X4753; Sigma-Aldrich, Sweden), an inverse ERR $\alpha$ agonist, for $32 \mathrm{~h}$. Cells were regularly tested to confirm the absence of mycoplasma contamination.

Small interfering RNAs (siRNAs) targeting PPARGCIA or ESRRA mRNA predesigned by ThermoFisher Scientific (Silencer Select; s21395 and s4830; Sweden) were used to silence the expression of these genes. Scramble siRNA (4390847; ThermoFisher Scientific) was used as negative control. Transfection of $\mathrm{C} 2 \mathrm{C} 12$ myoblasts and HSMCs was performed 3 and 6 days after induction of differentiation, respectively, in OptiMEM reduced serum media (31985062; ThermoFisher Scientific) with Lipofectamine RNAiMAX (13778; ThermoFisher Scientific).
Adenoviral delivery of human and mouse PPARGC1A (Ad-PGC1A) or $g f p$ (Ad-GFP) was performed overnight in differentiated HSMCs and $\mathrm{C} 2 \mathrm{C} 12$ myotubes. Experiments were performed $48 \mathrm{~h}$ after inducing transduction. In cells that were both treated with siRNA and transduced, silencing was performed as described above and cells were transduced immediately after transfection.

Mouse models Male mice were housed under controlled lighting (12 h light-dark cycle) with free access to food and water. Experiments were performed in accordance with Swiss federal guidelines and were approved by the Kantonales Veterinäramt Basel-Stadt. Skeletal muscle-specific Ppargcla transgenic mice (mTG) (C57BL/6-Tg(Ckm-Ppargcla)31Brsp/J; The Jackson Laboratory, USA; https://www.jax.org/strain/008231) are described elsewhere [19]. The Ppargcla muscle-specific knockout mice $(\mathrm{mKO})$ were generated by breeding PGC- $1 \alpha^{\text {loxP/loxP }}$ mice (B6N.129(FVB)-Ppargcla $a^{\mathrm{tm} 2.1 \mathrm{Brsp}} / \mathrm{J}$; The Jackson Laboratory; https://www.jax.org/strain/009666) [20] with transgenic mice expressing the Cre recombinase under the 
Table 2 Clinical characteristics of the participants included in the transcriptomic analysis

\begin{tabular}{|c|c|c|c|}
\hline Characteristic & NGT & Type 2 diabetes & $p$ value \\
\hline Age (years) & $58 \pm 1.8$ & $62 \pm 1.3$ & 0.0185 \\
\hline Weight (kg) & $84 \pm 1.7$ & $89 \pm 1.9$ & 0.0314 \\
\hline Height (m) & $1.80 \pm 0.02$ & $1.79 \pm 0.01$ & 0.7185 \\
\hline BMI $\left(\mathrm{kg} / \mathrm{m}^{2}\right)$ & $25.98 \pm 0.35$ & $27.94 \pm 0.48$ & 0.0020 \\
\hline Waist (cm) & $93 \pm 1.4$ & $101 \pm 1.7$ & 0.0020 \\
\hline Hip (cm) & $99 \pm 0.9$ & $101 \pm 1.3$ & 0.1691 \\
\hline WHR & $0.94 \pm 0.01$ & $0.99 \pm 0.01$ & 0.0009 \\
\hline Pulse (beats/min) & $61 \pm 1.4$ & $68 \pm 1.6$ & 0.0072 \\
\hline Fasting plasma glucose (mmol/l) & $5.46 \pm 0.08$ & $8.56 \pm 0.39$ & $<0.0001$ \\
\hline 120 min plasma glucose $(\mathrm{mmol} / \mathrm{l})$ & $5.89 \pm 0.27$ & $16.20 \pm 0.69$ & $<0.0001$ \\
\hline $\mathrm{HbA}_{1 \mathrm{c}}(\mathrm{mmol} / \mathrm{mol})$ & $35.76 \pm 0.64$ & $50.44 \pm 1.50$ & $<0.0001$ \\
\hline $\mathrm{HbA}_{1 \mathrm{c}}(\%)$ & $5.4 \pm 0.06$ & $6.8 \pm 0.14$ & $<0.0001$ \\
\hline Serum insulin (pmol/l) & $52.71 \pm 5.58$ & $92.58 \pm 9.01$ & 0.0008 \\
\hline 120 min serum insulin (pmol/l) & $314.9 \pm 50.8$ & $350.5 \pm 42.4$ & 0.5937 \\
\hline Plasma creatinine $(\mu \mathrm{mol} / \mathrm{l})$ & $83.64 \pm 2.36$ & $79.71 \pm 2.29$ & 0.2307 \\
\hline Plasma AST $(\mu k a t / l)$ & $0.38 \pm 0.03$ & $0.40 \pm 0.03$ & 0.7613 \\
\hline Plasma ALT ( $\mu$ kat/l) & $0.42 \pm 0.05$ & $0.50 \pm 0.04$ & 0.2565 \\
\hline Plasma triacylglycerol (mmol/l) & $1.25 \pm 0.15$ & $1.43 \pm 0.13$ & 0.4415 \\
\hline Plasma cholesterol (mmol/l) & $5.40 \pm 0.20$ & $4.48 \pm 0.15$ & 0.0005 \\
\hline Plasma HDL (mmol/l) & $1.30 \pm 0.05$ & $1.19 \pm 0.06$ & 0.1709 \\
\hline Plasma LDL (mmol/l) & $3.56 \pm 0.17$ & $2.63 \pm 0.15$ & 0.0002 \\
\hline Serum C-peptide (nmol/l) & $0.69 \pm 0.04$ & $1.03 \pm 0.08$ & 0.0008 \\
\hline HOMA-IR & $1.86 \pm 0.21$ & $5.23 \pm 0.64$ & $<0.0001$ \\
\hline
\end{tabular}

Results are shown as mean \pm SEM for individuals with NGT $(n=25)$ and type 2 diabetes $(n=25)$

Statistical analysis was performed using Student's $t$ test

ALT, alanine aminotransferase; AST, aspartate aminotransferase control of the human $\alpha$-skeletal actin promoter (B6.Cg$\operatorname{Tg}$ (ACTA1-cre)79Jme/J; The Jackson Laboratory; www.jax. org/strain/006149). Chow diet (AIN-93G; 7\% fat, 58.5\% carbohydrates, $18 \%$ protein and $16.5 \%$ of crude fibre, ash, and moisture) was provided by Provimi Kliba (Kaiseraugst, Switzerland).
Fig. 1 Flow chart illustrating participant enrolment and analysis. Sixty-one individuals were assessed for eligibility and six were excluded for not meeting the inclusion criteria. Group size for transcriptomic and metabolomic analysis is reported. Transcriptomic and metabolomic analysis shared samples from 13 individuals with NGT and 23 with type 2 diabetes

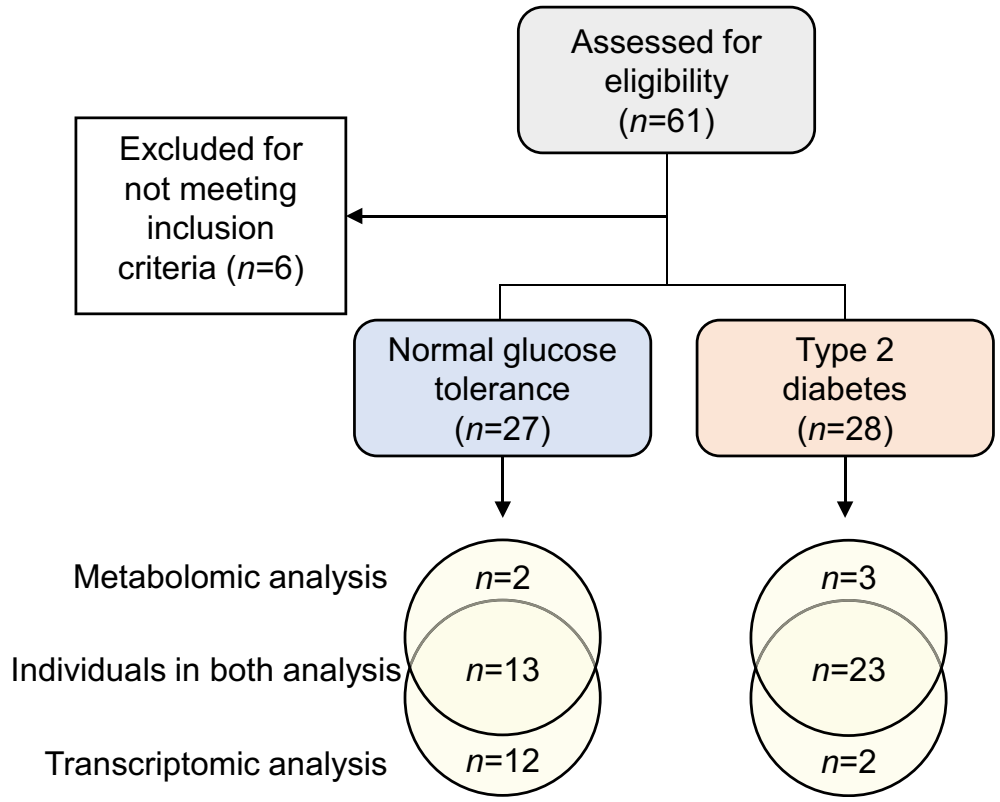


Fasted ( $4 \mathrm{~h}$ ) male mice aged 11-14 weeks were anaesthetised by intraperitoneal injection of pentobarbital $(150 \mathrm{mg} / \mathrm{kg})$ and tissues were collected. Metabolomic analysis of plasma and quadriceps muscle was performed at the Swedish Metabolomics Centre (Umeå University) as described [21]. See ESM Methods: Mouse plasma and skeletal muscle metabolomics for details.

Quantitative real-time RT-PCR Total RNA from human skeletal muscle biopsies, mouse skeletal muscles and cultured myotubes was extracted for mRNA analysis of BCAA genes. See ESM Methods: RNA isolation and relative mRNA expression for details. Primer sequences are shown in ESM Tables 1, 2.

Western blot analysis Equal amounts of protein from human skeletal muscle biopsies $(10 \mu \mathrm{g})$ and cells $(20 \mu \mathrm{g})$ were analysed for branched-chain $\alpha$-keto acid dehydrogenase (BCKDHA), $\mathrm{p}^{\mathrm{S} 293}$-BCKDHA, branched-chain keto acid dehydrogenase kinase (BCKDK) and protein phosphatase, $\mathrm{Mg}^{2+} / \mathrm{Mn}^{2+}$-dependent $1 \mathrm{~K}$ (PPM1K). See ESM Methods: Western blot analysis for details.

Leucine oxidation Myotube cultures were incubated with $1 \mathrm{ml}$ of Ham's F10 Medium in the presence of $0.1 \mathrm{mCi} / \mathrm{ml}$ radiolabelled leucine (L-[U- $\left.{ }^{14} \mathrm{C}\right]$ leucine; $11.1 \mathrm{GBq} / \mathrm{mmol}$, NEC279E250UC; PerkinElmer, MA, USA) and either 3,6dichlorobenzo(b)thiophene-2-carboxylic acid (BT2) (ENA018104907; Sigma-Aldrich) or DMSO. Small cups were placed in cell-culture wells and plates were air-tight sealed. After incubation for $4 \mathrm{~h}$ at $37^{\circ} \mathrm{C}, 150 \mu \mathrm{l}$ of $2 \mathrm{~mol} / \mathrm{l}$ $\mathrm{HCl}$ and $300 \mu \mathrm{l}$ of $2 \mathrm{~mol} / \mathrm{l} \mathrm{NaOH}$ were added into each well and small cup, respectively. After $1 \mathrm{~h}$, the liberated $\mathrm{CO}_{2}$ was collected and subjected to scintillation counting (Tri-Carb 4910TR; PerkinElmer).

Statistical analysis Results in tables are reported as means \pm SEM. Metabolites and gene expression results are reported as Tukey boxplots with median (line), $25-75 \%$ (box) and the 25 th $/ 75$ th percentile \pm 1.5 times the interquartile distance (whiskers). Values outside this range are plotted as individual open circles. Outlier values detected using the Grubb's test are plotted as closed circles and were not considered in the statistical analysis. HSMCs from each donor, $\mathrm{C} 2 \mathrm{C} 12$ myotubes at different passages, and single animals, were considered as experimental units. Randomisation and blinding were not carried out. Data normality was tested using the Shapiro-Wilk test. Homogeneity of variance was tested using the Levene's test. Data that did not meet these criteria were transformed with Tukey's ladder before the significance testing. Data were analysed using GraphPad Prism software (version 9.0.0; GraphPad Software, USA). Statistical tests and data information are indicated in the figure legends.
Fig. 2 Glucose loading further attenuates BCAA catabolism in individuals with type 2 diabetes. (a-I) Boxplots show scaled intensity values of BCAAs $(\mathbf{a}-\mathbf{c})$, BCKAs $(\mathbf{d}-\mathbf{f})$, BCAA-derived carnitines $(\mathbf{g}-\mathbf{k})$ and 3-hydroxyisobutyrate (l) in plasma and skeletal muscle from individuals with NGT $(n=15)$ or type 2 diabetes $(n=26)$, before (fasting) and after a 120 min OGTT. (m) Catabolic pathway of BCAAs and genes encoding involved enzymes (in orange boxes). BCKDH complex subunits are encoded by $B C K D H A, B C K D H B, D B T$ and $D L D$. Dashed arrows indicate intermediate metabolites (not shown). (n-p) Western blot analysis of BCKDHA and pBCKDHA ( $n=10$ per group). Expression values are expressed as relative to a control sample. Black circles represent outlier values. (q) Representative immunoblots of BCKDHA and pBCKDHA in fasting conditions (F) and after an OGTT (O). (r, s) Spearman correlation coefficients between plasma BCAA metabolites and clinical variables. Colour and size are proportional to correlation strength. (t-w) Examples of Spearman correlation coefficients summarised in $(\mathbf{r}, \mathbf{s})$. Statistical analysis was performed using two-way mixed-design ANOVA followed by Sidak's post hoc test. $* p<0.05, * * p<0.01$ and $* * * p<0.001 ;{ }^{\dagger} p<0.05,{ }^{\dagger} p<0.01$ and $\dagger^{\dagger} p<0.001$ vs NGT at the same feeding state. $\downarrow$, condition effect; $\S$, glucose loading effect; II, interaction effect. Main and interaction effects symbols indicate $p<0.05$ to $p<0.0001$. See also ESM Fig. 1 . $\beta$ Hydroxyisovaleryl, $\beta$-hydroxyisovalerylcarnitine; KIC, $\alpha$ ketoisocaproate; KIV, keto-isovaline; KMV, keto-methylvalerate, SKM, skeletal muscle; T2D, type 2 diabetes

A network graph showing top-ranked proteins interacting with PGC- $1 \alpha$ was generated using the STRING database (https://string-db.org/; accessed 18 May 2020) [22].

\section{Results}

Glucose loading further attenuates BCAA catabolism in individuals with type 2 diabetes Metabolomic profiling reveals that a glucose ingestion in fasted individuals elicits an insulindependent metabolic response, which is blunted in individuals with impaired glucose tolerance [23]. To assess whether this impaired response also affects the BCAA profile, we measured levels of leucine, isoleucine, valine and derived metabolites in plasma and vastus lateralis biopsies from individuals with either NGT or type 2 diabetes before and after an OGTT. Under fasted conditions, the three BCAAs were $\sim 10 \%$ and $\sim 13 \%$ higher in plasma and skeletal muscle, respectively, from individuals with type 2 diabetes as compared with NGT (Fig. 2a-c), and non-significant changes were found in the corresponding branched-chain $\alpha$-keto acids (BCKAs) (Fig. 2d-f). While BCAA-derived acylcarnitines were not different between groups (Fig. 2g-k), fasting 3-hydroxyisobutyrate (3HIB) was higher in plasma $(+37 \%)$ and skeletal muscle $(+45 \%)$ from individuals with type 2 diabetes as compared with NGT (Fig. 21). This valine-derived metabolite (Fig. 2m) promotes insulin resistance in skeletal muscle cells by increasing fatty acid uptake [24].

Glucose ingestion decreased circulating and intramuscular levels of BCAA and BCKA (Fig. 2a-f and ESM Fig. 1a,b), 

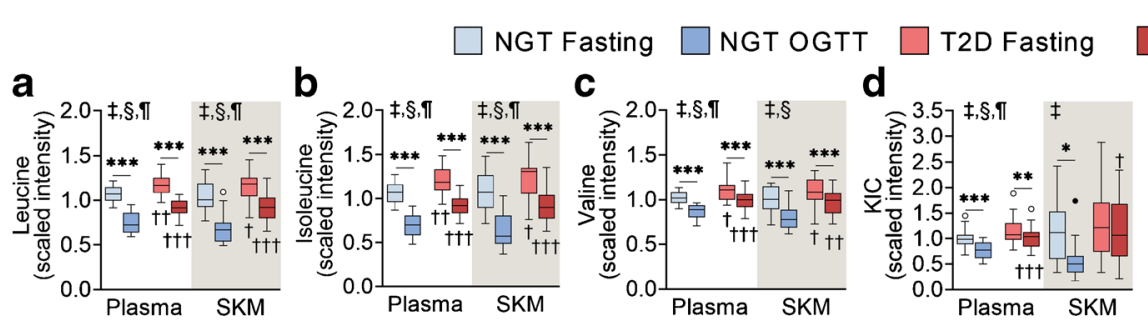

T2D OGTT
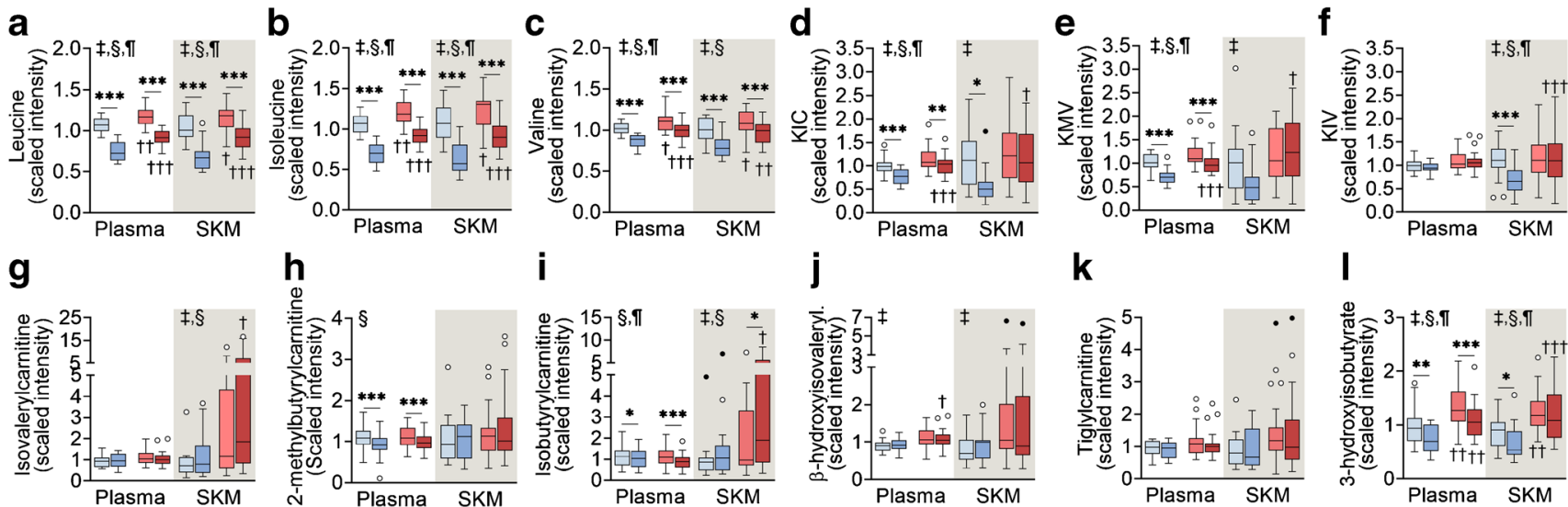

k

m

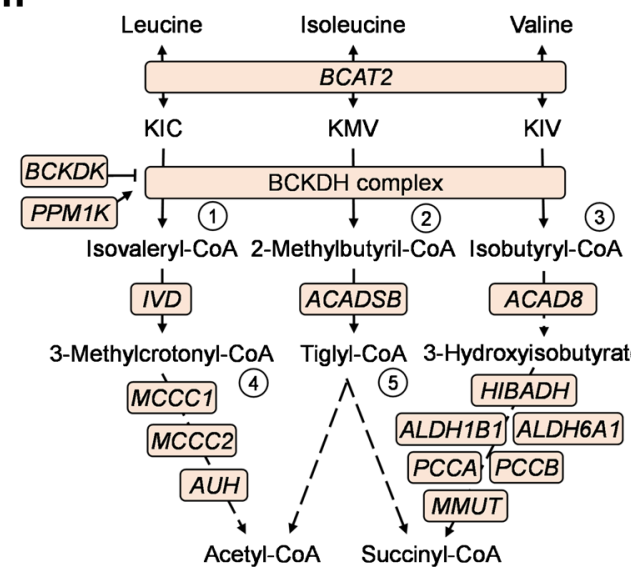

$\mathbf{r}$

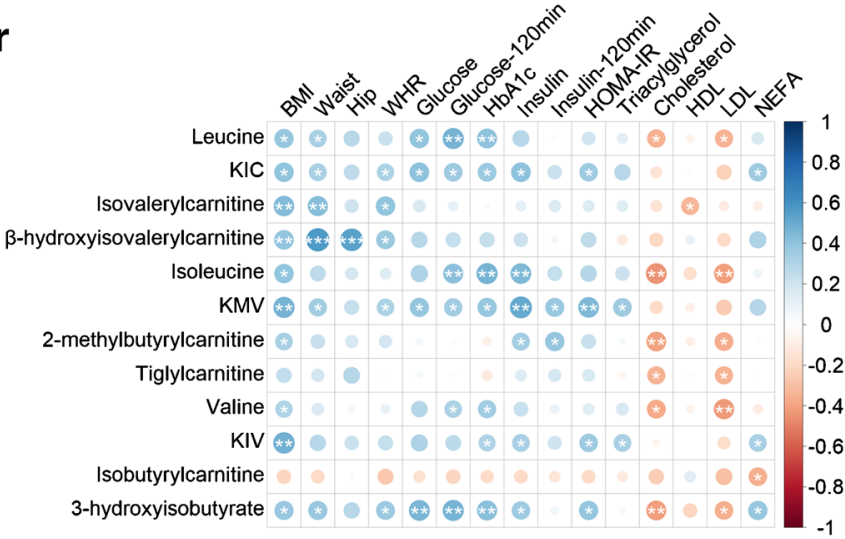

Plasma (Fasting)

t

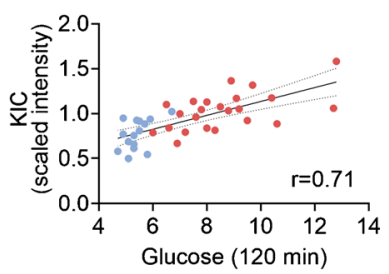

$\mathbf{u}$

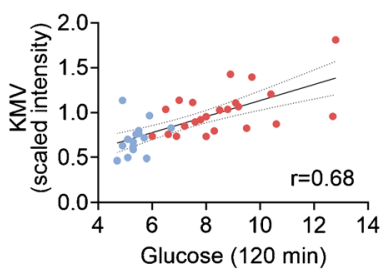

Derived carnitines:

(1) Isovalerylcarnitine

(2) 2-Methylbutyrylcarnitine

(3) Isobutyrylcarnitine

(4) $\beta$-hydroxyisovalerylcarnitine

(5) Tiglylcarnitine
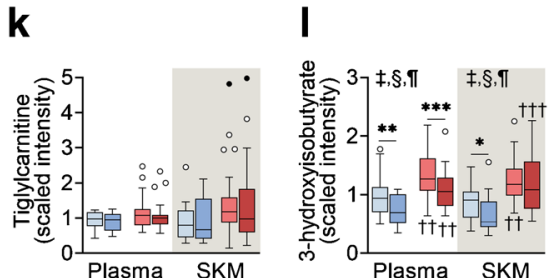

n
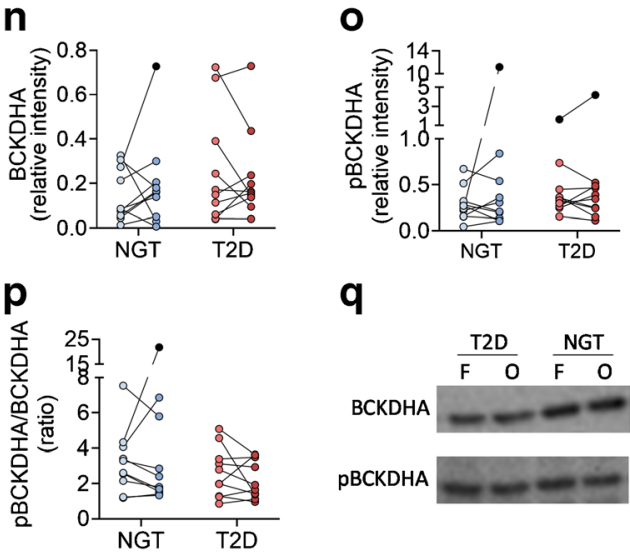

q

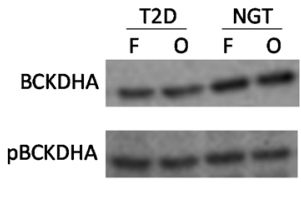

\section{$\mathbf{S}$}

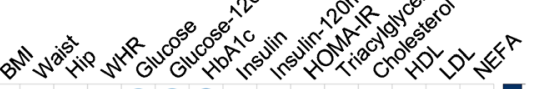

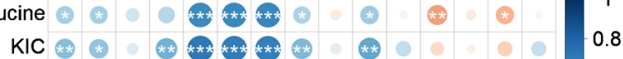

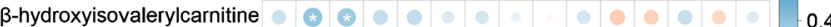

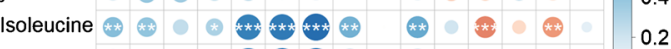

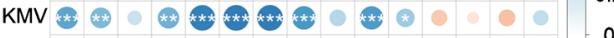

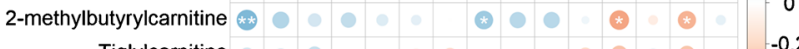

Tiglylcarnitine

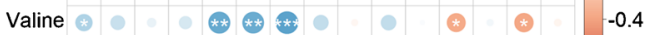

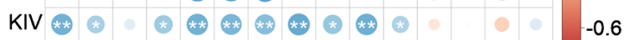

Isobutyrylcarnitine $0+0.8$

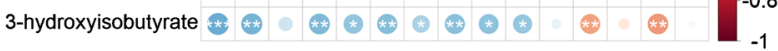

Plasma (after OGTT)

$\mathbf{V}$

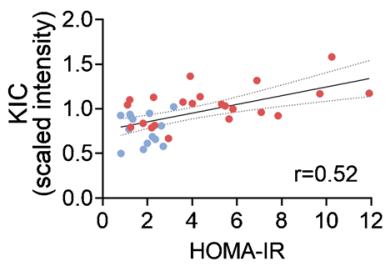

W

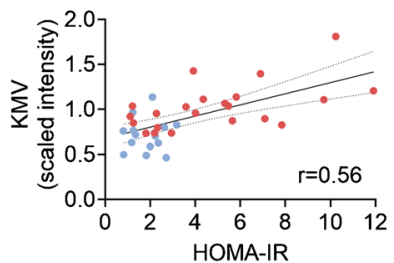


albeit to a lesser extent in individuals with type 2 diabetes. The intramuscular content of BCKA was decreased $37-56 \%$ in individuals with NGT, whereas levels remained unaltered in those with type 2 diabetes. These differences were not driven by the higher BMI and age in the type 2 diabetes group (ESM Table 3). BCKAs are irreversibly decarboxylated by the branched-chain keto acid dehydrogenase (BCKDH) complex, the rate-limiting enzyme in the catabolism of BCAA. We determined total abundance and phosphorylated (inactive) BCKDHA content in skeletal muscle (Fig. 2n-q) and found no differences between groups. Glucose loading decreased 3HIB levels in skeletal muscle of individuals with NGT but not type 2 diabetes (Fig. 21). Collectively, these results suggest that a glucose challenge unmasks defects at several steps of BCAA catabolism in type 2 diabetes. Indeed, circulating levels of leucine, isoleucine and derived BCKAs exhibited a positive correlation $(r=0.64-0.77)$ with blood glucose, $\mathrm{HbA}_{1 \mathrm{c}}$ levels and HOMA-IR after an OGTT (Fig. 2r-w and ESM Fig. 1c,d).

Expression of genes involved in BCAA catabolism is decreased in skeletal muscle from individuals with type 2 diabetes Expression of genes encoding enzymes involved in the first steps of BCAA metabolism, namely branched-chain amino acid transaminase 2 (BCAT2) and three subunits of the $\mathrm{BCKDH}$ complex were decreased in skeletal muscle of individuals with type 2 diabetes (Fig. 3a). Furthermore, 69\% of the analysed genes participating in metabolic steps downstream of BCKDH showed a similar profile (Fig. 3c), indicating that BCAA gene expression is widely downregulated in skeletal muscle of individuals with type 2 diabetes. These differences were also evident after an OGTT, as mRNA levels remained relatively stable. PGC- $1 \alpha$ is an upstream regulator of BCAA metabolism [15]. Expression of PPARGC1A, which encodes PGC- $1 \alpha$, was decreased in skeletal muscle of individuals with type 2 diabetes, irrespective of the feeding status (Fig. 3b). In addition, PPARGC1A was positively correlated with BCAA gene expression (ESM Fig. 2a) and BCAA metabolites in individuals with NGT but not type 2 diabetes (ESM Fig. 2b,c), while the expression of several BCAA genes was inversely associated with blood glucose and $\mathrm{HbA}_{1 \mathrm{c}}$ (Fig. $3 d)$. Expression of BCAA genes did not consistently correlate with BCAA-related metabolites (Fig. 3e) but exhibited opposite patterns in individuals with NGT and type 2 diabetes (ESM Fig. 2b,c).

PGC-1a mediates BCAA gene expression in primary HSMCs Primary HSMCs transfected with PPARGC1A siRNA displayed reduced expression of PPARGC1A (Fig. 4a). This transfection moderately decreased the expression of genes of the family of acyl-CoA dehydrogenases (ACAD8, ACADSB and $I V D$ ), and $H I B A D H$ (encoding the 3-H dehydrogenase enzyme), as compared with cells treated with a scrambled
siRNA (Fig. 4b). Adenovirus-mediated PPARGC1A overexpression (Ad-PGC1A) in HSMCs (Fig. 4c) increased the expression of $61 \%$ of the genes relative to adenovirus-GFP cells (Fig. 4d). We found increased PPM1K (Fig. 4f) and reduced $\mathrm{BCKDH}$ protein content in Ad-PGC1 A cells, associated with a higher pBCKDHA/BCKDHA ratio (Fig. 4g,i).

Mice with skeletal muscle-specific modified expression of Ppargc1a exhibit altered levels of BCAA gene transcripts and related metabolites Muscle-specific Ppargcla (encoding PGC-1 $\alpha$ ) knockout mice (mKO) had normal body weight (ESM Fig. 3a) and slightly impaired glucose tolerance (ESM Fig. 3b,c) as compared with wild-type (WT) littermates. Skeletal muscle from mKO mice (Fig. 5a) displayed decreased expression of most (67\%) of the analysed BCAA genes relative to WT littermates (Fig. 5b-d). Accordingly, a contrasting profile of BCAA gene expression was observed in skeletal muscle-specific Ppargcla transgenic mice (mTg) vs the mKO mice, with an upregulation of the BCAA genes relative to WT littermates (Fig. 5e-h). These changes in gene expression were not associated with alterations in either body weight or glucose tolerance (ESM Fig. 3d-f).

To ascertain whether PGC- $1 \alpha$-associated alterations in BCAA gene expression have functional implications in BCAA metabolism, we performed LC/MS metabolomics to profile BCAA-related metabolites in plasma and quadriceps muscle from $\mathrm{mKO}$ and $\mathrm{mTG}$ mice. Circulating BCAA levels in $\mathrm{mKO}$ and $\mathrm{mTG}$ mice were unaltered relative to respective WT littermate control mice (Fig. 5i-k), whereas the muscle content of valine was reduced in mTG mice (Fig. 51-n). Similar non-significant changes were observed for isoleucine $(p=0.09)$ and isovalerylcarnitine $(p=0.12)$ (Fig. $5 \mathrm{~m}, \mathrm{o})$, while mKO mice exhibited decreased levels of 3-HIB (Fig. 5q). Consistent with our results in HSMCs, C2C12 Ad-PGC1A upregulated the expression of BCAA-related genes (ESM Fig. $3 \mathrm{~g}$ ), and this was associated with increased leucine oxidation (ESM Fig. 3h).

PGC-1a regulates BCAA gene transcription through ERRa The orphan nuclear receptor $E R R \alpha$, encoded by ESRRA, is a canonical functional partner of PGC-1 $\alpha$ (Fig. 6a) that regulates metabolic processes in mitochondria [25]. Thus, we investigated whether ERR $\alpha$ is necessary for PGC- $1 \alpha-$ enhanced BCAA gene expression. siRNA-mediated silencing of ERR $\alpha$ (Fig. 6b) decreased the expression of $65 \%$ of the analysed genes (Fig. 6c). We determined whether this BCAA gene downregulation affects leucine oxidation in skeletal muscle. HSMCs were treated with either DMSO or BT2, a BCKDK inhibitor that increases BCAA oxidative flux. ESRRA silencing decreased leucine oxidation rates under BCKDH-activated conditions, as measured by $\mathrm{CO}_{2}$ production after incubation with $\left[\mathrm{U}_{-}{ }^{14} \mathrm{C}\right]$ leucine (Fig. $6 \mathrm{~d}, \mathrm{e}$ ). We next tested whether ERR $\alpha$ is a PGC- $1 \alpha$ interacting partner in the 
a

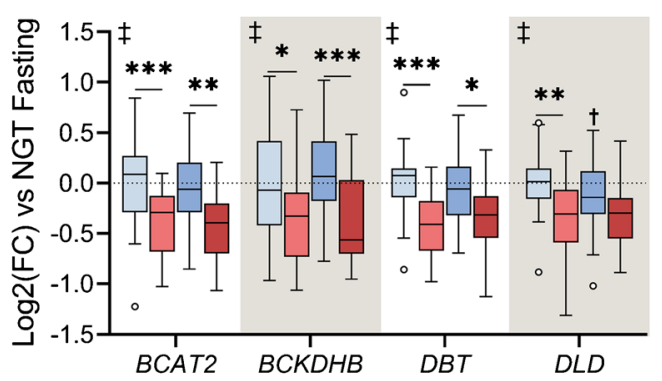

b

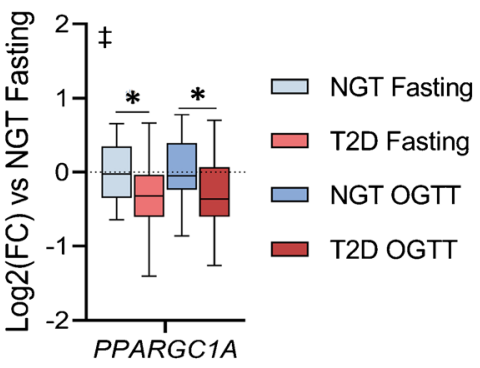

d

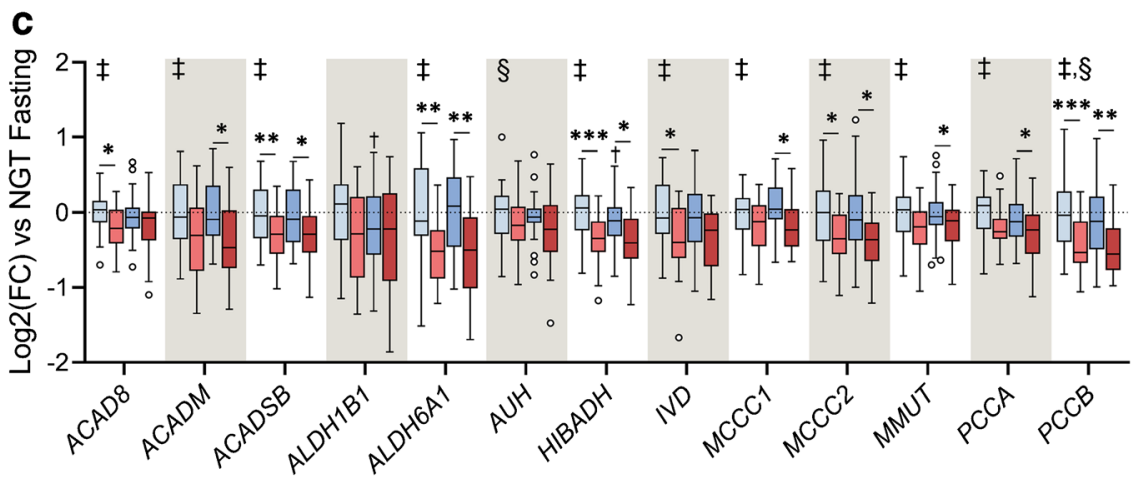

e

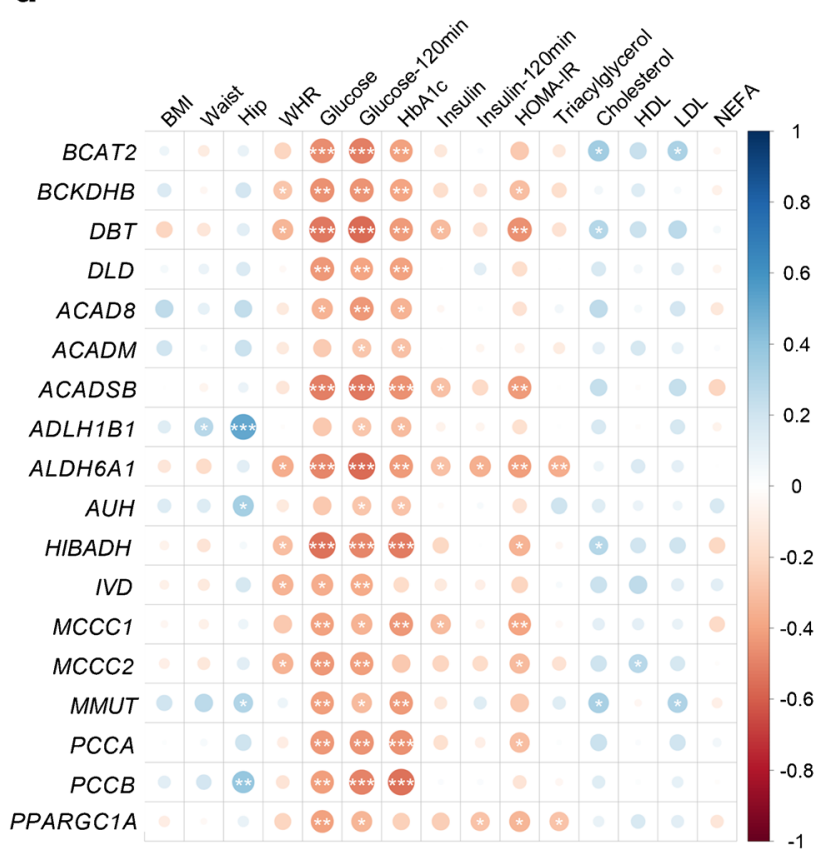

Fig. 3 Expression of genes involved in BCAA catabolism in individuals with type 2 diabetes and correlations with blood glucose levels. (a, c) BCAA gene expression in skeletal muscle biopsies of individuals with NGT $(n=25)$ or type 2 diabetes $(n=25)$ before and after a $120 \mathrm{~min}$ OGTT. (b) Expression of the transcriptional coactivator PPARGC1A. Gene expression is shown as $\log _{2}$ (fold change) relative to the NGT fasting group. Statistical analysis was performed using two-way mixeddesign ANOVA followed by Sidak's post hoc test. (d, e) Spearman

transcriptional regulation of the BCAA gene set using HSMCs treated with either scramble siRNA or ESRRA siRNA and transfected with Ad-GFP or Ad-PGC1A. We also

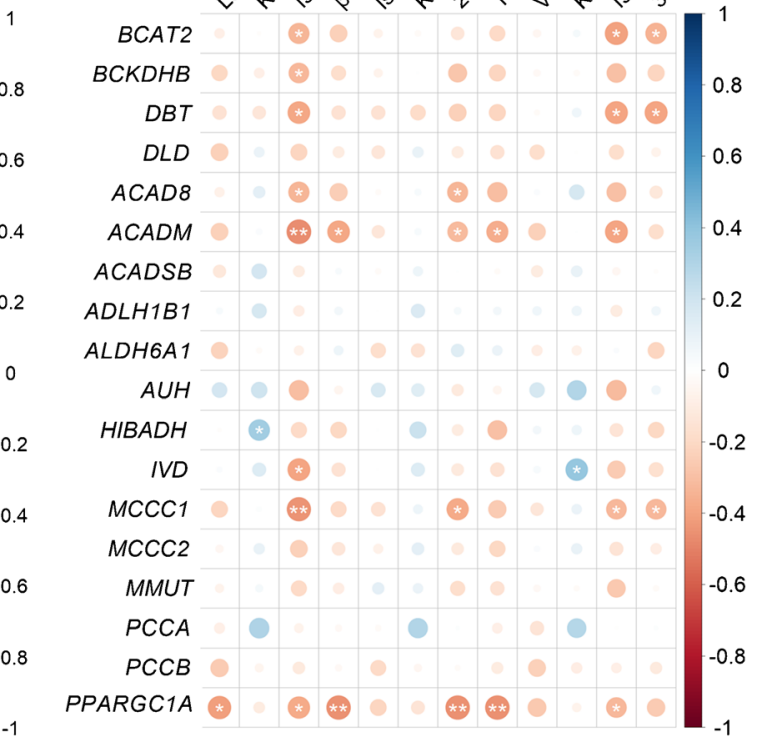

correlation coefficients between BCAA genes and clinical variables (d) or skeletal muscle BCAA metabolites (e) in fasted skeletal muscle. Colour and size are proportional to correlation strength. $* p<0.05$, $* * p<0.01$ and $* * * p<0.001 ; \dagger p<0.05$ vs NGT fasting. $\$$, condition effect; $\S$, glucose loading effect. Main and interaction effects symbols indicate $p<0.05$ to $p<0.0001$. See also ESM Fig. 2. FC, fold change; KIC, $\alpha$-ketoisocaproate; KIV, keto-isovaline; KMV, ketomethylvalerate; T2D, type 2 diabetes

tested this using Ad-PGC-1A cells treated with the inverse ERR $\alpha$ agonist XCT-790 (ESM Fig. 4). Since PPARGC1A and ESRRA mutually regulate their expression, we confirmed 


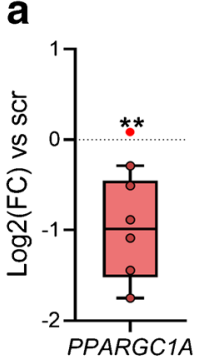

b
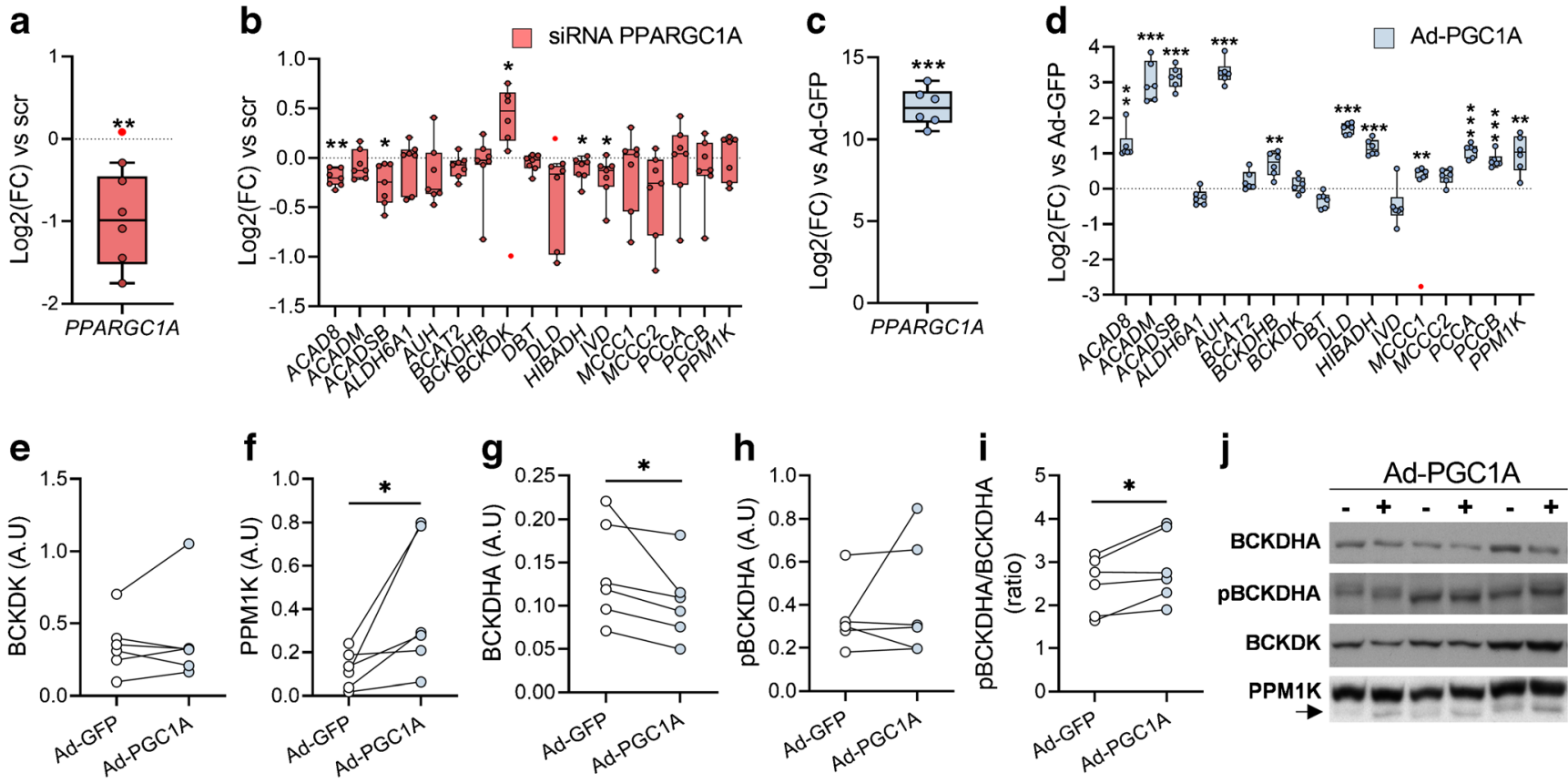

Fig. 4 PGC- $1 \alpha$ mediates BCAA gene expression in HSMCs. (a) Efficiency of PPARGC1A siRNA-mediated silencing. (b) Effects of the silencing of PPARGC1A on the expression of BCAA catabolic genes, $B C K D K$ and $P P M 1 K$. (c) Expression of PPARGC1A in Ad-PGC1A cells. (d) Effects of Ad-PGC1A on the expression of BCAA catabolic genes, $B C K D K$ and $P P M 1 K$. Gene expression is shown as $\log _{2}$ (fold change) relative to the corresponding scramble-treated cells (dotted line). (e-i)
Protein levels of BCKDK (e), PPM1K (f), BCKDHA (g) and phosphorylated BCKDHA (h), and the pBCKDHA/BCKDHA ratio (i). (j) Representative immunoblots. Arrow indicates the band corresponding to PPM1K protein. Statistical analysis was performed using paired $t$ test $(n=6) . * p<0.05, * * p<0.01$ and $* * * p<0.001$ vs scr $(\mathbf{a}, \mathbf{b})$ or Ad-GFP (c-i). Red circles indicate significant outliers not considered in statistical calculations. A.U, arbitrary units; FC, fold change; scr, scrambled siRNA that ESRRA silencing did not abrogate PPARGC1A in cultured cells. Ad-PGC1A cells had high levels of PPARGC1A transcripts regardless the treatment with ESRRA siRNA (Fig. 6f and ESM Fig. 4a), whereas the expression of two known targets of PGC- $1 \alpha /$ ERR $\alpha, T F A M$ and $V E G F$, was dampened by ESRRA siRNA despite the overexpression of PPARGC1A. Knockdown and inhibition of ESRRA completely ablated PGC-1 $\alpha$-mediated upregulation in all analysed genes (Fig. $6 \mathrm{~g}-\mathrm{i}$ and ESM Fig. $4 \mathrm{~b}-\mathrm{d}$ ), indicating that ERR $\alpha$ is essential for the transcriptomic regulation of the BCAA gene network orchestrated by PGC- $1 \alpha$. Nevertheless, ESRRA expression was similar between fasted individuals with either NGT or type 2 diabetes (Fig. 6j).

\section{Discussion}

A link between increased plasma BCAAs and insulin resistance was established as early as 1969 [3] but this association has remained relatively unexplored until the last decade. Here, we elucidate the mechanisms underpinning BCAA metabolism in type 2 diabetes. While we corroborate the association between BCAA metabolites and type 2 diabetes [9, 26-28] and the role of PGC- $1 \alpha$ in BCAA catabolism [15, 24, 29], we provide new evidence that an OGTT unmasks impairments in BCAA catabolism in individuals with type 2 diabetes. Moreover, we reveal that PGC- $1 \alpha$-mediated regulation of genes important for BCAA catabolism is dependent on $\mathrm{ERR} \alpha$, a canonical PGC- $1 \alpha$-interacting protein.

Circulating plasma BCAA levels are altered in individuals with type 2 diabetes; however, metabolomic profiles of peripheral tissues involved in glucose homeostasis are scarce and the transcriptomic regulation of genes involved in BCAA catabolism is unknown. Studies focusing on skeletal muscle BCAA content are limited to an analysis of adults with insulin resistance [14], rather than type 2 diabetes. We performed untargeted metabolomic analysis on both plasma and vastus lateralis biopsies obtained before and after an OGTT from men with either NGT or type 2 diabetes. Corroborating an earlier study of plasma samples from the Framingham Heart Study Offspring cohort [30], circulating BCAAs were decreased after an OGTT and this reduction was attenuated in type 2 diabetes. Concomitantly, we found that this blunted response also affected leucine- and isoleucine-derived BCKAs. In skeletal muscle, insulin inhibits proteolysis [31] and increases the preference for BCAA oxidation [13], which may explain the blunted excursion of BCAA and BCKA metabolites in insulin-resistant individuals with type 2 diabetes as compared with NGT. Consistent with this 

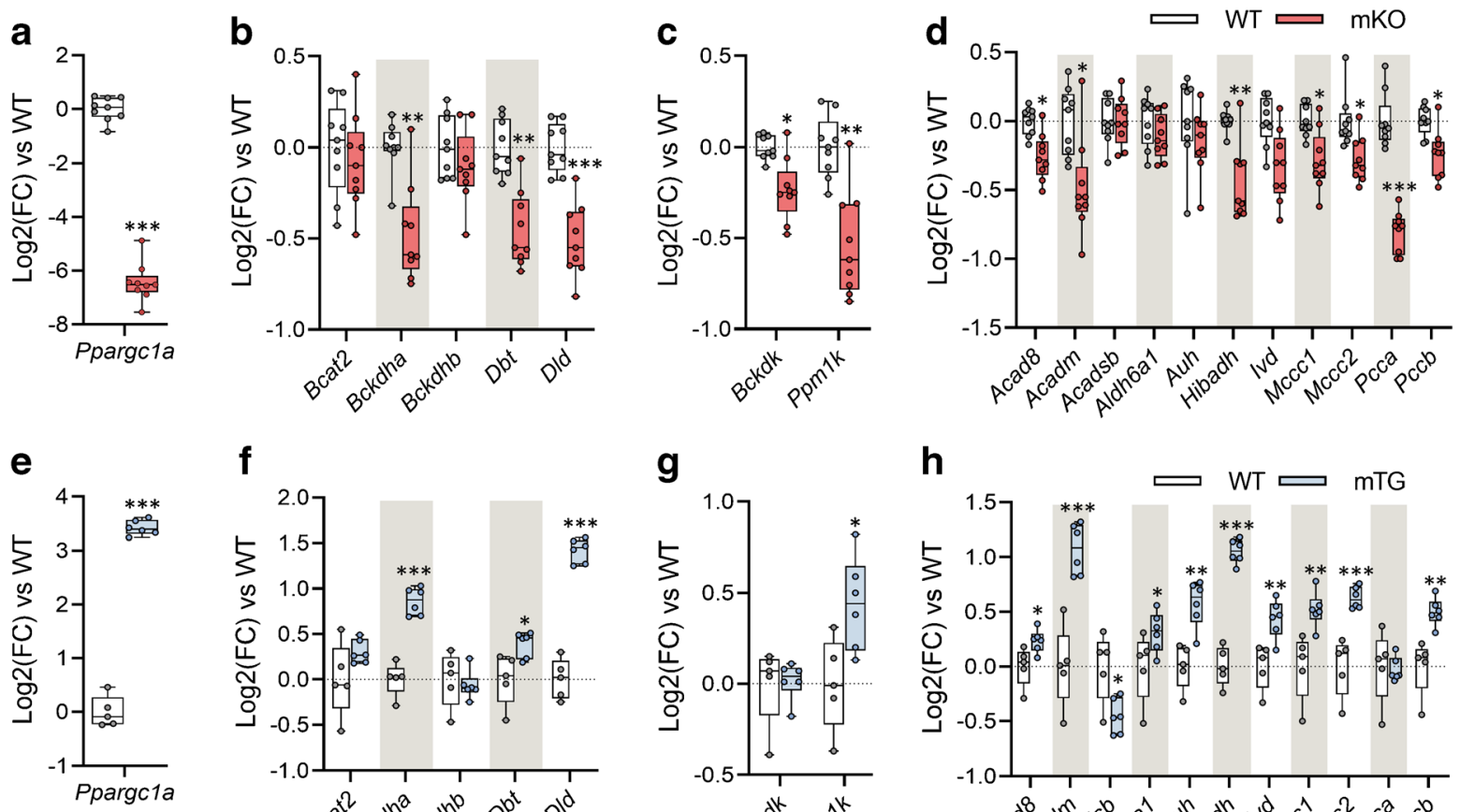

f

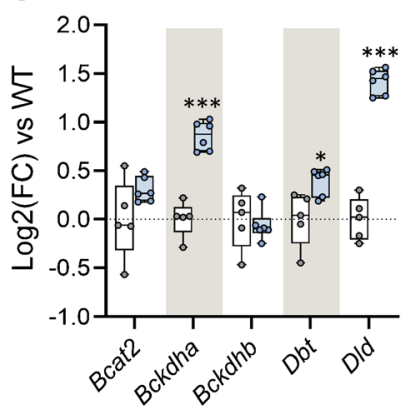

g

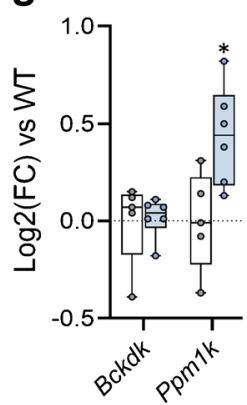

h

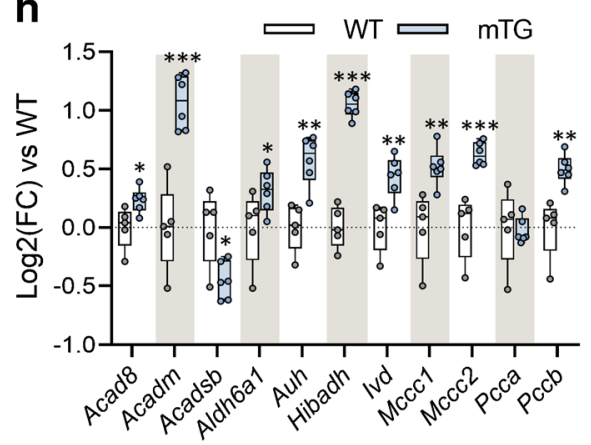

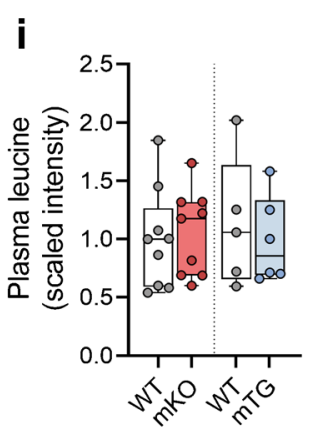

n

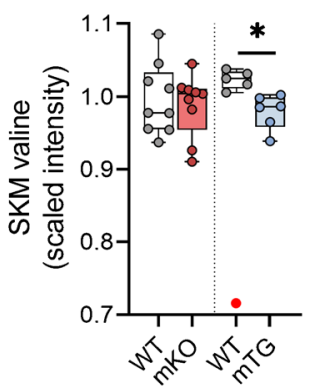

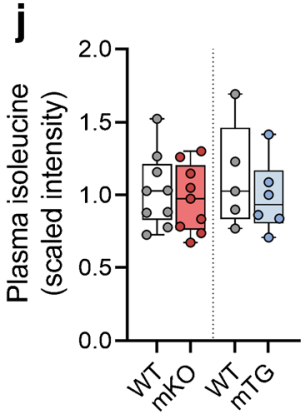

0

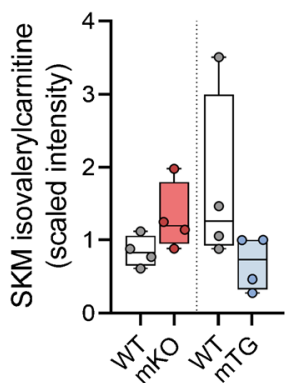

k

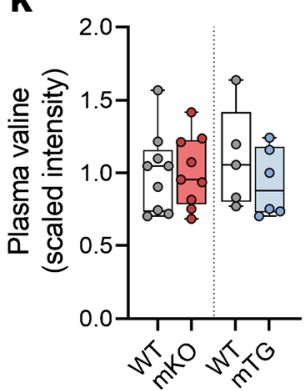

I

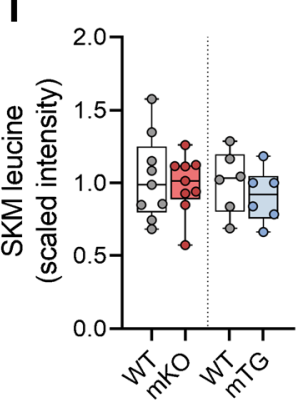

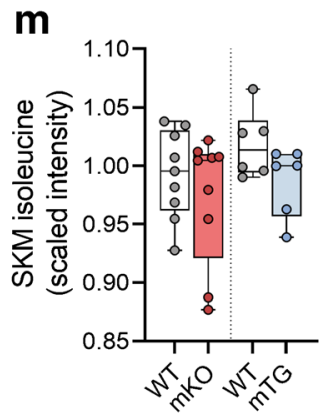

p

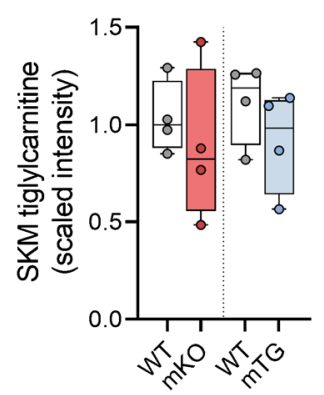

q

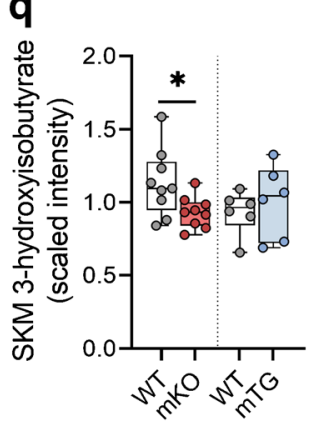

Fig. 5 Mice with skeletal muscle-specific modified expression of Ppargcla exhibit altered levels of BCAA gene transcripts and related metabolites. (a) Quadriceps Ppargcla expression in $\mathrm{mKO}$ mice and corresponding littermates $(n=9)$. (b-d) Expression of genes encoding BCAT2 and BCKDH subunits (b), BCKDK and PPM1K (c) and BCAA catabolic enzymes (d) in mKO mice. (e) Quadriceps Ppargcla expression in mTG mice $(n=6)$ and corresponding littermates $(n=5)$. $(\mathbf{f}-$ h) Expression of genes encoding BCAT2 and BCKDH subunits (f), BCKDK and PPM1K (g) and BCAA catabolic enzymes (h) in mTG mice. (i-k) Scaled intensity values of leucine, isoleucine and valine in plasma from $\mathrm{mKO}$ mice $(n=9)$, mTG mice $(n=6)$ and corresponding
WT littermates ( $n=9$ and $n=5$, respectively). (1-q) Scaled intensity values of BCAA and downstream intermediate metabolites in skeletal muscle from mKO mice $(n=9)$, mTG mice $(n=6)$ and corresponding WT littermates ( $n=9$ and $n=5$, respectively). Isovalerylcarnitine and tiglylcarnitine were measured in a subset of animals from each group $(n=4)$. Gene expression is shown as $\log _{2}$ (fold change) relative to WT mice. The dotted line represents the mean of the WT group. Statistical analysis was performed using unpaired $t$ test. $* p<0.05$, $* * p<0.01$ and $* * * p<0.001$ vs WT. Red circles indicate significant outliers not considered in statistical calculations. See also ESM Fig. 3. FC, fold change; SKM, skeletal muscle 
a

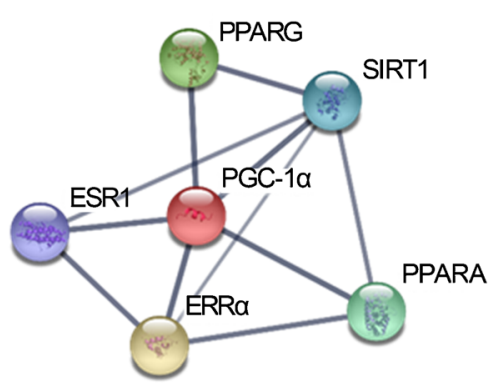

b

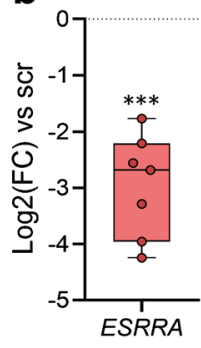

SIESRRA

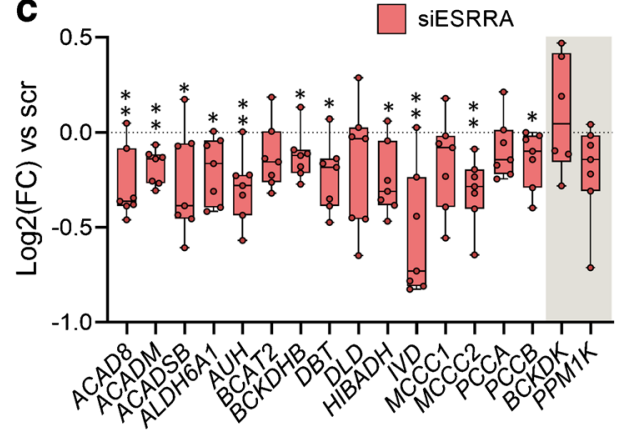

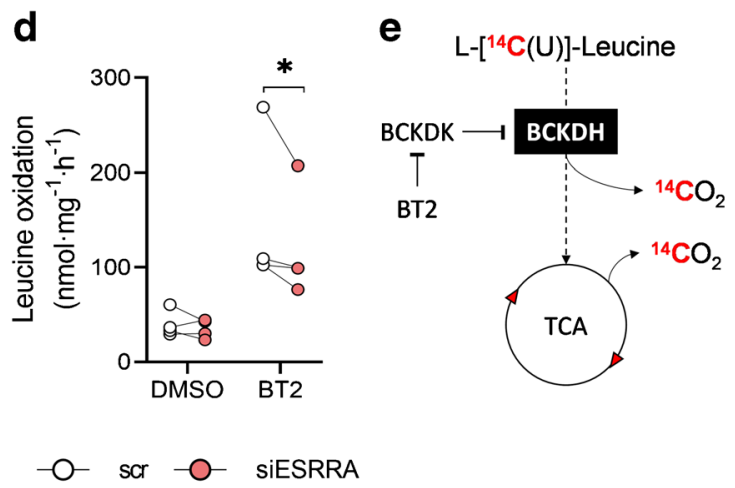
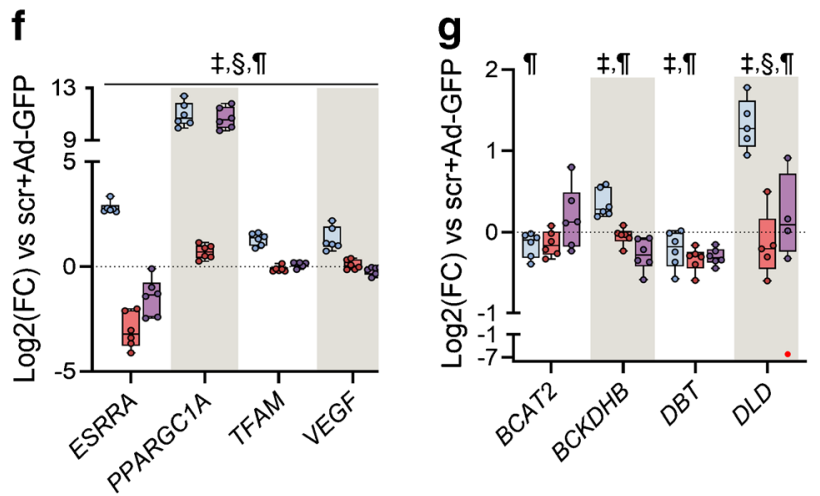
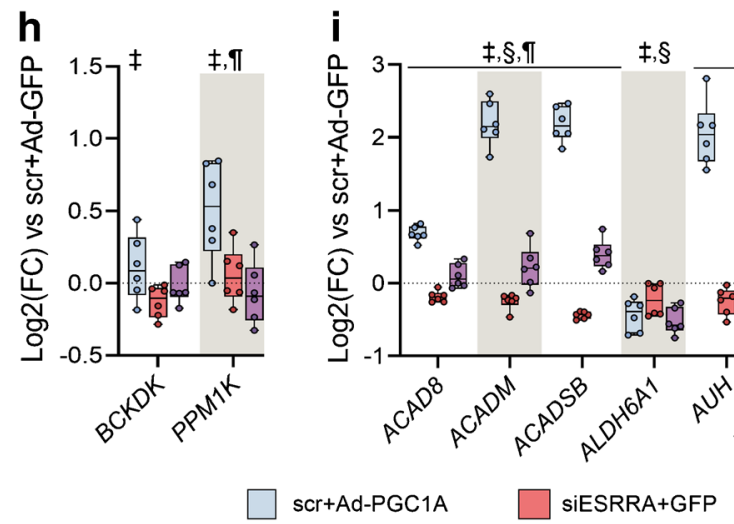

Fig. 6 PGC- $1 \alpha$ regulates BCAA gene transcription through ERR $\alpha$. (a) STRING Interacting Network showing the top five interacting proteins for PGC-1 $\alpha$. (b) Efficiency of ESRRA silencing in primary HSMCs. (c) Expression of BCAA genes. (d) Leucine oxidation rates in HSMCs. (e) Schematic biochemical representation of the leucine oxidation assay. (f) Expression of ESRRA, PPARGC1A and target genes in Ad-PGC1A cells transfected with ESRRA siRNA. (g-i) BCAA gene expression in AdPGC1A cells with or without ESRRA siRNA. (j) Expression of ESRRA in skeletal muscle biopsies from individuals with NGT or type 2 diabetes. Gene expression is shown as $\log _{2}$ (fold change) relative to the corresponding scramble+GFP treated cells (dotted line), scr (b, c), scr + Ad-GFP (f-

hypothesis, BCAAs, BCKAs and 3-HIB levels after the glucose challenge were significantly less decreased in the skeletal muscle from individuals with type 2 diabetes. Although we did not detect changes in BCKDHA phosphorylation state, BCAA catabolic flux cannot be predicted by BCKDH phosphorylation status [13], thus, we cannot exclude the possibility that BCKDH activity per se is impaired. Moreover, insulin modulates BCAA transamination in type 2 diabetes [32] and BCAT2 activity [33],

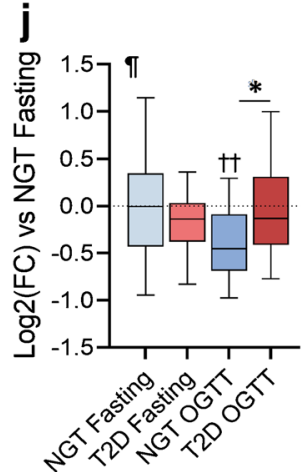

SiESRRA+Ad-PGC1A i) or NGT fasting (j). Statistical analysis was performed using paired $t$ test (b, c, $n=7$ ), two-way repeated measures ANOVA followed by Tukey's post hoc test (d, $n=4 ; \mathbf{f}-\mathbf{i}, n=6$, except in $\mathbf{g}: D L D, n=5)$, or two-way mixed-design ANOVA followed by Sidak's post hoc test $(\mathbf{j}, n=25)$. ${ }^{*} p<0.05, * * p<0.01$ and $* * * p<0.001$ vs scr $(\mathbf{b}, \mathbf{c})$ or scr-Ad-GFP (fi). $\$$, siESRRA effect; $\S$, Ad-PGC1A effect; II, interaction effect. Main and interaction effects symbols indicate $p<0.05$ to $p<0.0001$. See also ESM Fig. 4.; BT2, 3,6-dichlorobrenzo(b)thiophene-2-carboxylic acid; ESR1, oestrogen receptor 1; FC, fold change; scr, scrambled siRNA; SIRT1, sirtuin 1; TCA, tricarboxylic acid cycle

suggesting that insulin resistance could lead to an accumulation of BCKAs due to defects in both BCAA transamination and oxidation.

Generally, plasma metabolites mirrored the skeletal muscle BCAA profile. However, the intramuscular accumulation of isovalerylcarnitine, isobutyrylcarnitine and 3-HIB was not reflected in plasma, suggesting defects in processes controlling the export of metabolites from myocytes in type 2 
diabetes. Accordingly, the accumulation of 3-HIB after an OGTT is notable since this valine-derived metabolite promotes insulin resistance through increased fatty acid uptake in skeletal muscle [24]. This could lead to a vicious cycle in which secretion of 3-HIB from skeletal muscle may decrease insulin sensitivity and further impair insulin signalling. Although elevated levels of circulating C3 and C5 acylcarnitines have been also detected in individuals with obesity [7], whether accumulation of short-chain acylcarnitines mediates in insulin resistance remains to be elucidated [34]. These results suggest that the degradation of BCAA-derived metabolites is incomplete. Additionally, we found a strong correlation of post-OGTT BCAAs and derived BCKA circulating levels with blood glucose and $\mathrm{HbA}_{1 \mathrm{c}}$. Since plasma fasting BCAA levels exhibited a much weaker relationship with clinical biomarkers of type 2 diabetes, this finding underscores a tight connection between impaired glucose homeostasis and whole-body BCAA catabolism.

The accumulation of BCAA metabolites in individuals with type 2 diabetes was accompanied by reduced expression of genes involved in BCAA catabolism, suggesting that alterations in the transcriptional regulation of these genes could attenuate BCAA catabolism in skeletal muscle. Contrasting with previous findings [35], we did not find a correlation between BCAA genes and related metabolites, suggesting that post-transcriptional modifications play a key role in the impairment of BCAA catabolism. Conversely, the BCAA gene set was inversely correlated with blood glucose levels, suggesting a connection between glucose homeostasis and alterations in the transcriptional regulation of genes involved in BCAA catabolism.

PGC- $1 \alpha$ mediates the expression of genes involved in BCAA catabolism [15, 24, 29]. Concordantly, expression of PPARGC1A was positively correlated with BCAA genes. PGC- $1 \alpha$ also coordinates metabolic and transcriptomic programs linked to cellular energy homeostasis [36-38], and reduced PGC- $1 \alpha$ mRNA and protein levels are linked to insulin resistance in type 2 diabetes [39, 40]. In the present study, primary HSMCs and mouse skeletal muscle with reduced PPARGC1A expression exhibited a mild reduction in expression of several BCAA genes, whereas PPARGC1A overexpression was associated with a consistent upregulation. Thus, while PGC- $1 \alpha$ was not essential for basal BCAA gene transcription, a role in the adaptive response to increased BCAA catabolic demands, such as during exercise or nutritional changes, cannot be excluded [41]. We next hypothesised that alterations in the BCAA gene network would impact BCAA metabolism. We found that mice overexpressing Ppargcla in skeletal muscle exhibited an upregulation of BCAA genes and reduced intramuscular accumulation of valine, suggestive of increased BCAA catabolic flux. Similarly, adenovirus-mediated overexpression of Ppargcla in $\mathrm{C} 2 \mathrm{C} 12$ myotubes increased leucine oxidation rates. However, intramuscular levels of BCAA metabolites were unaltered in $\mathrm{mKO}$ mice. Thus, other organs such as adipose tissue may compensate for a putative impairment in BCAA catabolism [42]. Furthermore, a metabolic challenge may be necessary to reveal functional alterations in BCAA metabolism in $\mathrm{mKO}$ and $\mathrm{mTG}$ mice.

PGC- $1 \alpha$ is a transcriptional coactivator and does not directly bind DNA in a sequence-specific manner. The nuclear orphan receptor ERR $\alpha$ is one of the main transcriptional partners of PGC- $1 \alpha$ [25], and through this physical interaction, elicits the transcription of genes involved in mitochondrial biogenesis and oxidative phosphorylation [43], lipid metabolism [44] and ketone body homeostasis [45]. We found that ESRRA silencing, as well as inhibition of ERR $\alpha$ with an inverse agonist, downregulated BCAA genes and abrogated the PGC- $1 \alpha$-induced responses, indicating that ERR $\alpha$ is necessary for the PGC- $1 \alpha$-mediated transcription of BCAA genes. Moreover, ESRRA silencing was associated with a modest decrease in BCKDH-stimulated leucine oxidation, suggesting a functional impact of the transcriptional downregulation of BCAA genes under energy-demanding conditions. Thus, we next hypothesised that alterations in ERR $\alpha$ may explain the defects in BCAA catabolism in the individuals with type 2 diabetes. However, expression of ESRRA was unaltered in skeletal muscle of individuals with NGT or type 2 diabetes, suggesting that reduction in PGC- $1 \alpha$ is sufficient to impair BCAA expression or, alternatively, the PGC- $1 \alpha$-ERR $\alpha$ interaction is compromised in type 2 diabetes.

Some limitations of our study warrant discussion. Our analysis was confined to male participants and therefore we cannot exclude sex-specific differences in the analysed outcomes. Most of the individuals with type 2 diabetes included in this study were treated with metformin. Considering that mitochondria are the major target of metformin, this may affect BCAA metabolism. Due to limitations in the LC/MS methodology, quantification of BCAA metabolites with CoA moieties was not possible. To overcome this, we used specific derived carnitines from these metabolites as a proxy. Alterations in PGC- $1 \alpha$ expression may influence fibre type distribution $[19,46]$, which could impact the results. Nevertheless, similar results were obtained in transgenic mice, in vitro primary HSMCs and $\mathrm{C} 2 \mathrm{C} 12$ myotubes, suggesting that alterations in BCAA metabolism are unrelated to fibre type.

In conclusion, altered expression of PPARGC1A is associated with disturbances in BCAA metabolism in skeletal muscle of individuals with type 2 diabetes. Experimental approaches to reduce PPARGC1A levels partially recapitulates the BCAA gene set profile identified in skeletal muscle from individuals with type 2 diabetes, without impacting circulating and intramuscular BCAA levels. Our results indicate that glucose loading exacerbates disturbances in the BCAA profile, revealing that the metabolic inflexibility that characterises type 2 diabetes encompasses BCAA catabolism. Additionally, our data demonstrate that ERR $\alpha$ is essential for PGC- $1 \alpha$-mediated transcriptional regulation of genes involved in BCAA metabolism in primary human myotubes, 
thereby unravelling a new role for this orphan nuclear receptor.

Supplementary Information The online version contains peer-reviewed but unedited supplementary material available at https://doi.org/10.1007/ s00125-021-05481-9.

Data availability The datasets generated during and/or analysed during the current study are available from the corresponding author on reasonable request.

Funding Open access funding provided by Karolinska Institutet. The cost of the human metabolomics study was supported by a grant from Daiichi Sankyo Co., Ltd. This work was supported by a grant from the Novo Nordisk Foundation (NNF17OC0030088), Swedish Diabetes Foundation (DIA2018-357), Swedish Research Council (2015-00165, 2018-02389), the Strategic Research Programme in Diabetes at Karolinska Institutet (2009-1068) and the Knut and Alice Wallenberg Foundation (2018-0094). DRR is supported by a Novo Nordisk postdoctoral fellowship run in partnership with Karolinska Institutet.

Authors' relationships and activities JH is employee of Daiichi Sankyo Co. All other authors declare that there are no relationships or activities that might bias, or be perceived to bias, their work.

Contribution statement RJOS conceived the idea, planned the experiments and collected and analysed data. DRR performed leucine oxidation assays, analysed data and wrote the manuscript. AVC contributed to discussion and interpretation of metabolomic and protein data. EC collected mouse metabolite data and contributed to discussion. RF assisted with animal care, OGTT and skeletal muscle sampling. SK and $\mathrm{JH}$ contributed to the conception of the study. HKRK assisted with recruitment and collection of human metabolite data. $\mathrm{CH}$ provided mouse biological samples and contributed to the discussion of animal data. TM supervised acquisition and analysis of mouse metabolite data. AK contributed to the conception and design of the study and contributed to the discussion of all results. EN assisted with recruitment and obtained human skeletal muscle biopsies and blood samples. JRZ contributed to the conception and design of the study, edited the manuscript and acquired funding. All authors have reviewed the article and gave their final approval. JRZ is the guarantor of this work and, as such, had full access to all the data in the study and takes responsibility for the integrity of the data and the accuracy of the data analysis.

Open Access This article is licensed under a Creative Commons Attribution 4.0 International License, which permits use, sharing, adaptation, distribution and reproduction in any medium or format, as long as you give appropriate credit to the original author(s) and the source, provide a link to the Creative Commons licence, and indicate if changes were made. The images or other third party material in this article are included in the article's Creative Commons licence, unless indicated otherwise in a credit line to the material. If material is not included in the article's Creative Commons licence and your intended use is not permitted by statutory regulation or exceeds the permitted use, you will need to obtain permission directly from the copyright holder. To view a copy of this licence, visit http://creativecommons.org/licenses/by/4.0/.

\section{References}

1. Leahy JL (2005) Pathogenesis of type 2 diabetes mellitus. Arch Med Res 36(3):197-209. https://doi.org/10.1016/j.arcmed.2005. 01.003
2. Galgani JE, Moro C, Ravussin E (2008) Metabolic flexibility and insulin resistance. Am J Physiol Endocrinol Metab 295(5):E1009E1017. https://doi.org/10.1152/ajpendo.90558.2008

3. Felig P, Marliss E, Cahill GF Jr (1969) Plasma amino acid levels and insulin secretion in obesity. N Engl J Med 281(15):811-816. https://doi.org/10.1056/NEJM196910092811503

4. Nie C, He T, Zhang W, Zhang G, Ma X (2018) Branched chain amino acids: beyond nutrition metabolism. Int J Mol Sci 19(4):954. https://doi.org/10.3390/ijms19040954

5. Patti ME, Brambilla E, Luzi L, Landaker EJ, Kahn CR (1998) Bidirectional modulation of insulin action by amino acids. J Clin Invest 101(7):1519-1529. https://doi.org/10.1172/JCI1326

6. Huffman KM, Shah SH, Stevens RD et al (2009) Relationships between circulating metabolic intermediates and insulin action in overweight to obese, inactive men and women. Diabetes Care 32(9):1678-1683. https://doi.org/10.2337/dc08-2075

7. Newgard CB, An J, Bain JR et al (2009) A branched-chain amino acid-related metabolic signature that differentiates obese and lean humans and contributes to insulin resistance. Cell Metab 9(4):311326. https://doi.org/10.1016/j.cmet.2009.02.002

8. Wurtz P, Soininen P, Kangas AJ et al (2013) Branched-chain and aromatic amino acids are predictors of insulin resistance in young adults. Diabetes Care 36(3):648-655. https://doi.org/10.2337/dc120895

9. Menni C, Fauman E, Erte I et al (2013) Biomarkers for type 2 diabetes and impaired fasting glucose using a nontargeted metabolomics approach. Diabetes 62(12):4270-4276. https://doi.org/10. 2337/db13-0570

10. Shah SH, Crosslin DR, Haynes CS et al (2012) Branched-chain amino acid levels are associated with improvement in insulin resistance with weight loss. Diabetologia 55(2):321-330. https://doi. org/10.1007/s00125-011-2356-5

11. Lotta LA, Scott RA, Sharp SJ et al (2016) Genetic predisposition to an impaired metabolism of the branched-chain amino acids and risk of type 2 diabetes: a Mendelian randomisation analysis. PLoS Med 13(11):e1002179. https://doi.org/10.1371/journal.pmed.1002179

12. Fukagawa NK, Minaker KL, Rowe JW et al (1985) Insulinmediated reduction of whole body protein breakdown. Doseresponse effects on leucine metabolism in postabsorptive men. $\mathrm{J}$ Clin Invest 76(6):2306-2311. https://doi.org/10.1172/JCI112240

13. Neinast MD, Jang C, Hui S et al (2019) Quantitative analysis of the whole-body metabolic fate of branched-chain amino acids. Cell Metab 29(2):417-429 e414. https://doi.org/10.1016/j.cmet.2018. 10.013

14. Lerin C, Goldfine AB, Boes $\mathrm{T}$ et al (2016) Defects in muscle branched-chain amino acid oxidation contribute to impaired lipid metabolism. Mol Metab 5(10):926-936. https://doi.org/10.1016/j. molmet.2016.08.001

15. Hatazawa Y, Tadaishi M, Nagaike Y et al (2014) PGC-1alphamediated branched-chain amino acid metabolism in the skeletal muscle. PLoS One 9(3):e91006. https://doi.org/10.1371/journal. pone. 0091006

16. van Doorn M, Vogels J, Tas A et al (2007) Evaluation of metabolite profiles as biomarkers for the pharmacological effects of thiazolidinediones in type 2 diabetes mellitus patients and healthy volunteers. Br J Clin Pharmacol 63(5):562-574. https://doi.org/10. 1111/j.1365-2125.2006.02816.x

17. Evans AM, DeHaven CD, Barrett T, Mitchell M, Milgram E (2009) Integrated, nontargeted ultrahigh performance liquid chromatography/electrospray ionization tandem mass spectrometry platform for the identification and relative quantification of the small-molecule complement of biological systems. Anal Chem 81(16):6656-6667. https://doi.org/10.1021/ac901536h

18. Al-Khalili L, Chibalin AV, Kannisto K et al (2003) Insulin action in cultured human skeletal muscle cells during differentiation: 
assessment of cell surface GLUT4 and GLUT1 content. Cell Mol Life Sci 60(5):991-998. https://doi.org/10.1007/s00018-003-30013

19. Lin J, Wu H, Tarr PT et al (2002) Transcriptional co-activator PGC1 alpha drives the formation of slow-twitch muscle fibres. Nature 418(6899):797-801. https://doi.org/10.1038/nature00904

20. Lin J, Wu PH, Tarr PT et al (2004) Defects in adaptive energy metabolism with CNS-linked hyperactivity in PGC-1alpha null mice. Cell 119(1):121-135. https://doi.org/10.1016/j.cell.2004.09. 013

21. Chorell E, Ryberg M, Larsson C et al (2016) Plasma metabolomic response to postmenopausal weight loss induced by different diets. Metabolomics 12(5):1-14. https://doi.org/10.1007/s11306-0161013-x

22. Szklarczyk D, Gable AL, Lyon D et al (2019) STRING v11: protein-protein association networks with increased coverage, supporting functional discovery in genome-wide experimental datasets. Nucleic Acids Research 47(D1). https://doi.org/10.1093/ nar/gky1131

23. Shaham O, Wei R, Wang TJ et al (2008) Metabolic profiling of the human response to a glucose challenge reveals distinct axes of insulin sensitivity. Mol Syst Biol 4:214. https://doi.org/10.1038/ msb.2008.50

24. Jang C, Oh SF, Wada S et al (2016) A branched-chain amino acid metabolite drives vascular fatty acid transport and causes insulin resistance. Nat Med 22(4):421-426. https://doi.org/10.1038/nm. 4057

25. Vernier M, Giguere V (2021) Aging, senescence and mitochondria: the PGC-1/ERR axis. J Mol Endocrinol 66(1):R1-R14. https://doi. org/10.1530/JME-20-0196

26. Suhre K, Meisinger C, Doring A et al (2010) Metabolic footprint of diabetes: a multiplatform metabolomics study in an epidemiological setting. PLoS One 5(11):e13953. https://doi.org/10.1371/journal. pone. 0013953

27. Xu F, Tavintharan S, Sum CF, Woon K, Lim SC, Ong CN (2013) Metabolic signature shift in type 2 diabetes mellitus revealed by mass spectrometry-based metabolomics. J Clin Endocrinol Metab 98(6):E1060-E1065. https://doi.org/10.1210/jc.2012-4132

28. Yu D, Moore SC, Matthews CE et al (2016) Plasma metabolomic profiles in association with type 2 diabetes risk and prevalence in Chinese adults. Metabolomics 12:3 https://doi.org/10.1007/ s11306-015-0890-8

29. Hatazawa Y, Minami K, Yoshimura R et al (2016) Deletion of the transcriptional coactivator PGClalpha in skeletal muscles is associated with reduced expression of genes related to oxidative muscle function. Biochem Biophys Res Commun 481(3-4):251-258. https://doi.org/10.1016/j.bbrc.2016.10.133

30. Ho JE, Larson MG, Vasan RS et al (2013) Metabolite profiles during oral glucose challenge. Diabetes 62(8):2689-2698. https:// doi.org/10.2337/db12-0754

31. Meek SE, Persson M, Ford GC, Nair KS (1998) Differential regulation of amino acid exchange and protein dynamics across splanchnic and skeletal muscle beds by insulin in healthy human subjects. Diabetes 47(12):1824-1835. https://doi.org/10.2337/diabetes.47. 12.1824

32. Halvatsiotis P, Short KR, Bigelow M, Nair KS (2002) Synthesis rate of muscle proteins, muscle functions, and amino acid kinetics in type 2 diabetes. Diabetes 51(8):2395-2404. https://doi.org/10. 2337/diabetes.51.8.2395

33. David J, Dardevet D, Mosoni L, Savary-Auzeloux I, Polakof S (2019) Impaired skeletal muscle branched-chain amino acids catabolism contributes to their increased circulating levels in a non-obese insulin-resistant fructose-fed rat model. Nutrients 11(2):355 https:// doi.org/10.3390/nu1 1020355

34. Schooneman MG, Vaz FM, Houten SM, Soeters MR (2013) Acylcarnitines: reflecting or inflicting insulin resistance? Diabetes 62(1):1-8. https://doi.org/10.2337/db12-0466

35. Hernández-Alvarez MI, Díaz-Ramos A, Berdasco M et al (2017) Early-onset and classical forms of type 2 diabetes show impaired expression of genes involved in muscle branched-chain amino acids metabolism. Sci Rep 7(1):13850. https://doi.org/10.1038/s41598017-14120-6

36. Estall JL, Kahn M, Cooper MP et al (2009) Sensitivity of lipid metabolism and insulin signaling to genetic alterations in hepatic peroxisome proliferator-activated receptor-gamma coactivator1alpha expression. Diabetes 58(7):1499-1508. https://doi.org/10. 2337/db08-1571

37. Wu Z, Puigserver P, Andersson U et al (1999) Mechanisms controlling mitochondrial biogenesis and respiration through the thermogenic coactivator PGC-1. Cell 98(1):115-124. https://doi.org/10. 1016/S0092-8674(00)80611-X

38. Yoon JC, Puigserver P, Chen G et al (2001) Control of hepatic gluconeogenesis through the transcriptional coactivator PGC-1. Nature 413(6852):131-138. https://doi.org/10.1038/35093050

39. Moreno-Santos I, Perez-Belmonte LM, Macias-Gonzalez M et al (2016) Type 2 diabetes is associated with decreased PGC1alpha expression in epicardial adipose tissue of patients with coronary artery disease. J Transl Med 14(1):243. https://doi.org/10.1186/ s12967-016-0999-1

40. Patti ME, Butte AJ, Crunkhorn S et al (2003) Coordinated reduction of genes of oxidative metabolism in humans with insulin resistance and diabetes: potential role of PGC1 and NRF1. Proc Natl Acad Sci U S A 100(14):8466-8471. https://doi.org/10.1073/pnas. 1032913100

41. Hagg SA, Morse EL, Adibi SA (1982) Effect of exercise on rates of oxidation, turnover, and plasma clearance of leucine in human subjects. Am J Phys 242(6):E407-E410. https://doi.org/10.1152/ ajpendo.1982.242.6.E407

42. Herman MA, She P, Peroni OD, Lynch CJ, Kahn BB (2010) Adipose tissue branched chain amino acid (BCAA) metabolism modulates circulating BCAA levels. J Biol Chem 285(15):1134811356. https://doi.org/10.1074/jbc.M109.075184

43. Schreiber SN, Emter R, Hock MB et al (2004) The estrogen-related receptor alpha (ERRalpha) functions in PPARgamma coactivator 1 alpha (PGC-1alpha)-induced mitochondrial biogenesis. Proc Natl Acad Sci U S A 101(17):6472-6477. https://doi.org/10.1073/pnas. 0308686101

44. Luo J, Sladek R, Carrier J, Bader JA, Richard D, Giguere V (2003) Reduced fat mass in mice lacking orphan nuclear receptor estrogenrelated receptor alpha. Mol Cell Biol 23(22):7947-7956. https:// doi.org/10.1128/mcb.23.22.7947-7956.2003

45. Svensson K, Albert V, Cardel B, Salatino S, Handschin C (2016) Skeletal muscle PGC-1alpha modulates systemic ketone body homeostasis and ameliorates diabetic hyperketonemia in mice. FASEB J 30(5):1976-1986. https://doi.org/10.1096/fj.201500128

46. Handschin C, Chin S, Li P et al (2007) Skeletal muscle fiber-type switching, exercise intolerance, and myopathy in PGC-1alpha muscle-specific knock-out animals. J Biol Chem 282(41):30014 30021. https://doi.org/10.1074/jbc.M704817200

Publisher's note Springer Nature remains neutral with regard to jurisdictional claims in published maps and institutional affiliations. 